Portland State University

PDXScholar

$1-1-2010$

\title{
Vertical Noise Structure and Target Detection Performance in Deep Ocean Environments
}

Zizheng Li

Portland State University

Follow this and additional works at: https://pdxscholar.library.pdx.edu/open_access_etds Let us know how access to this document benefits you.

\section{Recommended Citation}

$\mathrm{Li}$, Zizheng, "Vertical Noise Structure and Target Detection Performance in Deep Ocean Environments" (2010). Dissertations and Theses. Paper 138.

https://doi.org/10.15760/etd.138

This Thesis is brought to you for free and open access. It has been accepted for inclusion in Dissertations and Theses by an authorized administrator of PDXScholar. Please contact us if we can make this document more accessible: pdxscholar@pdx.edu. 
Vertical Noise Structure and Target Detection Performance in

Deep Ocean Environments

\author{
by \\ Zizheng Li
}

A thesis submitted in partial fulfillment of the requirements for the degree of

\author{
Master of Science \\ in \\ Electrical and Computer Engineering
}

Thesis Committee:

Lisa M. Zurk, Chair

Martin Siderius

Branimir Pejcinovic

Portland State University

(C) 2010 


\begin{abstract}
In passive sonar systems, knowledge of low-frequency shipping noise is an important factor for target detection performance. However, an accurate model for the shipping noise structure is difficult to obtain, due to the varying distributions of ships and complicated underwater environment. This work characterizes low-frequency distant shipping noise observed in deep water environments as a function of receiver depth and vertical arrival structure for the case of a receiver below the conjugate depth. Surface shipping noise is examined using Monte Carlo simulations using a normal mode propagation model based on random distribution of ships and realistic parameters. The depth dependence of the simulated distant shipping noise is in agreement with published experimental measurements. A Vertical Line Array (VLA) is used to produce vertical beams that isolate the surface interference from nearby targets. Simulation results quantifying the beamformer output as a function of ocean environment, receiver aperture, and frequency are presented for both conventional and adaptive beamformers. The results suggest that conventional beamforming could detect the noisy target from both direct arrival and bottom bounce in the presence of distant shipping interferers and wind noise. However, the beamwidth of conventional beamforming is wider than that of adaptive beamforming. Once the motion effects of nearby ship interferences are considered, the adaptive
\end{abstract}


beamforming using diagonal loading provides better detection performance. Preliminary adaptive beamforming results corresponding to different snapshot times show that motion effects can be minimized by using short observation times. 


\section{Acknowledgments}

I would like to acknowledge and thank my advisor, Dr. Lisa Zurk, for all of her help and support during this research. Her patience and encouragement have been invaluable. I also thank Dr. Martin Siderius who provided useful input and encouragement, and I appreciate Dr. Brano who served on my thesis committee. I would like to thank the students in the NEAR-Lab for their help. I am also grateful to my family who gave me lots of support. In addition, I would like to acknowledge the Office of Naval Research for funding this work. 


\section{Contents}

$\begin{array}{ll}\text { Abstract } & \text { i }\end{array}$

Acknowledgments

List of Tables vi vi

List of Figures vii

1 Introduction 1

1.1 Contributions of This Work ............... 2

1.2 Background Overview . . . . . . . . . . . . . . . . 2

1.3 Sonar System . . . . . . . . . . . . . . . . . . . 6

1.3.1 Basic Concepts ................. 6

1.3.2 The Sonar Equation . . . . . . . . . . . . . . . 7

1.4 Underwater Acoustic Wave Propagation and Normal Modes . . . 8

2 Signal and Noise Models $\quad 15$

2.1 Historical Temporal Shipping (HITS) database . . . . . . . . . 15

2.2 Source Level and Source Depth . . . . . . . . . . . . . . 18 
2.3 Wind Noise Model . . . . . . . . . . . . . . . . . 20

3 Detection Performance $\quad 22$

3.1 Array Gain . . . . . . . . . . . . . . . . 22

3.2 Beamforming Techniques . . . . . . . . . . . . . 23

3.2.1 Conventional Beamforming ............ 25

3.2 .2 Adaptive Beamforming . . . . . . . . . . . . . 28

3.3 Source Motion Analysis . . . . . . . . . . . . . . . . . . . 32

4 Simulation Results and Analysis 35

4.1 Simulation Process . . . . . . . . . . . . . . . . 35

4.1.1 Simulation Conditions . . . . . . . . . . . . 35

4.1.2 Monte Carlo Simulation Procedures . . . . . . . . . . . . 39

4.2 Simulation Results and Analysis . . . . . . . . . . . . . . . 41

4.2.1 Vertical Arrival Structure of Shipping Noise . . . . . . . 43

4.2.2 Beamforming and Directionality for the VLA . . . . . . 48

4.2 .3 Motion Effect Analysis . . . . . . . . . . . . 53

5 Conclusions and Future Work $\quad 58$

$\begin{array}{ll}\text { References } & 60\end{array}$ 


\section{List of Tables}

2.1 Source Depth (SD) and ANDES Source Level Model for Different Ship Types . . . . . . . . . . . . . . . . . 20

2.2 Sea State v.s. Source Strength and Wind Speed at $50 \mathrm{~Hz}$. . . . . 21

4.1 Simulated Bottom Condition in Church Opal . . . . . . . . . . 38

4.2 Source Level of the Target Classification . . . . . . . . . . . . . . . 39 


\section{List of Figures}

1.1 Flow chart of KRAKEN normal mode program. . . . . . . . . . . 11

1.2 Transmission loss for the Munk profile with a source depth and frequency of $100 \mathrm{~m}$ and $50 \mathrm{~Hz}$, respectively, simulated by KRAKEN normal mode program including (a) 63 modes (waterborne modes only), and (b) 102 modes (waterborne modes and bottom bounce modes). . . . . . . . . . . . . . . . . 13

1.3 The Munk sound-speed profile. . . . . . . . . . . . . . . . . 14

2.1 Average deep-water ambient noise spectra. Figure taken from $\operatorname{Urick}[10] \ldots \ldots \ldots \ldots \ldots \ldots$

2.2 Global merchants distribution in December, 1998. Figure is generated based on HITS database. . . . . . . . . . . . . . . . . 18

2.3 ANDES source level model [13]. The source level spectra calculated by ANDES model depend only on ship class, not on ship length or speed. . . . . . . . . . . . . . . . 19 
3.1 A narrowband beamformer forms a linear combination of the sensor outputs [17]. The outputs of sensors are each multiplied by a complex weight then summed. . . . . . . . . . . . . . . 24

3.2 Comparison between conventional and adaptive beamforming at $50 \mathrm{~Hz}$. Two sources are at 30 degrees and 35 degrees, with $40 \mathrm{~dB}$ source level. One weak source is at 0 degree with $10 \mathrm{~dB}$ source level. The array is 10 elements with spacing of half wavelength, and white noise is $10 \mathrm{~dB}$. Adaptive beamforming with MVDR could resolve the two closed sources and detect the weak source, while conventional beamforming could not. . . . . . . . . 30

3.3 Source motion diagram. The interferer is moving towards the VLA. (a) The interferer moves a very short distance due to low moving speed or short time duration. In this case, the moving interferer can be still considered as one source. (b) The interferer moves in a long distance. In this case, the moving interferer might look like multiple sources. . . . . . . . . . . . . . . 33

4.1 Locations of CHURCH OPAL measurement sites [1] . . . . . 36

4.2 SSP at Church Opal in September, from Gaul et al [1] . . . . . 37

4.3 VLA for beamforming simulations. . . . . . . . . . . 39 
4.4 Comparison between the statistics from HITS database and Monte Carlo realizations in September: Statistics Data from HITS database for (a) large tankers and (c) fishing vessels; the average data of 5000 Monte Carlo realizations for (b) large tankers and (d) fishing vessels. The cyan triangle is the location of the VLA. . . . . 42

4.5 Transmission loss at $50 \mathrm{~Hz}$ for a super tanker calculated using KRAKEN propagation normal mode with 329 modes. The SSP and boundary conditions are given in Figure 4.2 and Table 4.1, respectively. . . . . . . . . . . . . . . . . 43

4.6 Simulated noise level at different sea states at $50 \mathrm{~Hz}$. The source strengths for these 3 sea states are $45 \mathrm{~dB}, 59 \mathrm{~dB}$, and $68 \mathrm{~dB}$ respectively. Wind speeds are 4-6 knots, 11-16 knots and 28-33 knots respectively. . . . . . . . . . . . . . . . . 44

4.7 Distribution of the received levels from distant surface shipping for a receiver located at different depths at $50 \mathrm{~Hz}$ : The depths for the receiver are $50 \mathrm{~m}$ (near surface), $600 \mathrm{~m}$ (sound channel axis), $4060 \mathrm{~m}$ (critical depth), and $4850 \mathrm{~m}$ (near bottom), respectively. The received level near the bottom is the smallest. The received levels for VLA located near surface and located at critical depth have similar pattern. . . . . . . . . . . . . . . 45 
4.8 Simulated ambient noise levels as a function of depth at (a) $50 \mathrm{~Hz}$ and (b) $300 \mathrm{~Hz}$. Ambient noise includes surface distant shipping noise and wind noise. The wind noises correspond to $5 \mathrm{kn}, 10$ $\mathrm{kn}$ and $15 \mathrm{kn}$, respectively. The VLA is located near the bottom from $3430 \mathrm{~m}$ to $4850 \mathrm{~m} \ldots \ldots \ldots$. . . . . . . . . 46

4.9 Measured ambient noise levels as a function of depth at (a) 50 $\mathrm{Hz}$ and (b) $300 \mathrm{~Hz}$. Figures are taken from Gaul [1]. . . . . . . . 46

4.10 Computed bottom transmission loss as a function of sediment thickness. (a) Simulation geometry. The receiver is located at the depth of $4850 \mathrm{~m}, 30 \mathrm{~m}$ above the bottom. The source depth is $10 \mathrm{~m}$. The source to receiver ranges are $200 \mathrm{~km}, 800 \mathrm{~km}, 1500 \mathrm{~km}$ and $3000 \mathrm{~km}$, respectively. (b) Computed bottom transmission loss as a function of sediment thickness at $50 \mathrm{~Hz}$ for a layer of silty clay sediment. . . . . . . . . . . . . . . 47

4.11 Conventional beamforming with $45 \mathrm{~dB}$ Chebyshev Taper at 50 Hz. The target is at $5 \mathrm{~km}$ range, $100 \mathrm{~m}$ depth. Noise includes distant shipping noise, $5 \mathrm{kn}$ wind noise and $20 \mathrm{~dB}$ white noise. Target source levels are (a) $115 \mathrm{~dB}$ (average source level) and (b) $137 \mathrm{~dB}$ (noisy source level). . . . . . . . . . . . . . . 49 
4.12 Same as Figure 4.11 with a higher frequency of $300 \mathrm{~Hz}$. Target source levels are (a) $100 \mathrm{~dB}$ (average source level), and (b) 120 $\mathrm{dB}$ (noisy source level). . . . . . . . . . . . . . . 5 50

4.13 Adaptive beamforming without diagonal loading at $300 \mathrm{~Hz}$. The target is at $5 \mathrm{~km}$ range, $100 \mathrm{~m}$ depth. Noise includes distant shipping noise, $5 \mathrm{kn}$ wind noise and $20 \mathrm{~dB}$ white noise. Target source levels are (a) $100 \mathrm{~dB}$ (average source level), and (b) 120 $\mathrm{dB}$ (noisy source level). . . . . . . . . . . . . . . 51

4.14 Adaptive beamforming with diagonal loading at $300 \mathrm{~Hz}$. Diagonal loading level is $25 \mathrm{~dB}$. Other parameters are the same as Figure $4.13 \ldots \ldots \ldots \ldots \ldots$. . . . . . . . . . . . . . . 52

4.15 Computed nearby ships based on HITS database at the location of Church Opal. The colorbar shows the total ship number in 5000 realizations. For example, at the cell with source to receiver range of $20 \mathrm{~km}$, there are about 4 ships in all during 5000 realizations. The nearby ship based on the realistic data has minimal effects as interferers. . . . . . . . . . . . . 54

4.16 Change of source to receiver range during 60 snapshots. The snapshot length are 0.1 seconds and 10 seconds, respectively. . . 55 
4.17 Eigenvalue spectrum structure of the moving interferer covariance matrix. The snapshot length is (a) 0.1 seconds and (b) 10

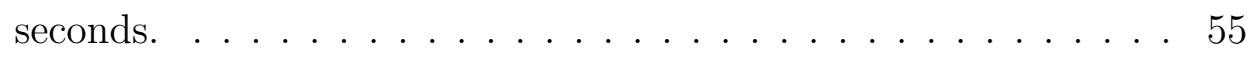

4.18 Adaptive beamforming output with $25 \mathrm{~dB}$ diagonal loading at $300 \mathrm{~Hz}$. The target is at $5 \mathrm{~km}$ range, $100 \mathrm{~m}$ depth. Noise includes all shipping noise, $5 \mathrm{kn}$ wind noise, $20 \mathrm{~dB}$ white noise and noise from the moving large tanker. $\mathrm{L}=60$ Snapshots. The interval times between snapshots are (a) 0.1 seconds, and (b) 10 seconds. 57 


\section{Chapter 1}

\section{Introduction}

In previous research done by Gaul et al. [1], the measurements of ambient noise due to distant surface ships at the location of Church Opal, NE pacific, show the depth dependence of distant shipping noise. In this research, these measurements are used as a basis for developing a prediction model to understand the nature of ambient noise in deep ocean environments. The inputs for this model are the ship distribution at the area of interest, the environment parameters (including sound speed profile, the characteristic of seabed and sediment conditions, etc), and the depths of sources and receivers. The output will give the received shipping noise in the specific area. From the simulation results, the ambient noise levels can be successfully modeled in complicated deep water environments. In addition, the measurement results indicate that the distant shipping noise can be significantly reduced when measured with an array located below the critical depth ${ }^{1}$. With the vertical noise structure predicted by the model, target detection performance, which has military and civilian significance, can be improved by using beamforming techniques with a vertical line array.

\footnotetext{
${ }^{1}$ Critical depth is defined as the depth in the deep isothermal layer at which the sound speed is the same as it is at the surface [2].
} 


\subsection{Contributions of This Work}

The work presented here makes the following contributions:

- Produced a model to simulate the ambient noise in deep ocean environments. This model can be used to analyze surface shipping noise in any area of interest.

- Demonstrated depth dependence of the noise at phones in and below the critical depth, which is in agreement with experimental measurements in Gaul [1]. Simulation results show that the distant shipping noise can be significantly reduced when measured with the receivers located below the critical depth, which can be applied to improve target detection performance.

- Developed beamforming techniques on target detection in the presence of distant shipping noise and wind noise in the deep ocean environments. In addition, this work also analyzed nearby interference source motion effect.

\subsection{Background Overview}

Research on low-frequency shipping noise structure for target detection in the ocean environment has both military and civilian applications [3]. Shipping noise dominates the ambient noise field in the 50-300 $\mathrm{Hz}$ frequency band. In the deep ocean environment, distant shipping noise has a broad peak around $30 \mathrm{~Hz}$ and quickly declines above $100 \mathrm{~Hz}$ [4]. 
Noise arising from shipping can be modeled as emanating from point sources at precise locations, or by shipping densities on a latitude and longitude grid [5]. The Historical Temporal Shipping (HITS) database is a global database of surface shipping density, and can be used as an input to propagation models to describe the levels and directional dependence of the low-frequency ambient noise as a function of time and location. A Vertical Line Array (VLA) is usually utilized to produce vertical beams to isolate the surface interference from nearby targets. Noise levels received from the VLA at a specific position depend on the acoustic propagation conditions between the VLA and the surface ships. Wind noise received by the VLA in the deep ocean environment typically has steep angles from the upward vertical [5].

To improve target detection performance in the presence of surface shipping noise and wind noise, it is necessary to characterize the ambient noise. In passive sonar, beamforming is utilized to extract the angular information in the form of beams. Conventional beamforming is inadequate due to its limited sidelobe rejection [6]. Adaptive beamforming is therefore used to improve detection performance. Adaptive beamforming is typically based on the minimum variance distortionless response (MVDR) formulation [7]. One major cause of performance degradation in adaptive beamformers is mismatch loss due to incorrect steering vectors. When the steering vector is perfectly known, 
adaptive beamforming can give high spatial resolution and good interference suppression, but when the presumed steering vector and actual steering vector are different, an adaptive beamformer suppresses target signals, often referred to as the self-nulling phenomenon. In this work, diagonal loading is applied to improve the robustness of the adaptive beamformer [8].

At low frequencies, the ambient noise is dominated by loud surface ships that move through many narrow beams. The process of obtaining a satisfactory sample covariance matrix is inefficient in this case due to the lack of a stationary environment. Recent trends in passive sonar systems include the application of large-aperture arrays with hundreds of sensors to form narrow beams in order to detect a weak target in the presence of fast-moving, strong interferers. Such large arrays typically require more snapshots and longer duration snapshots due to the increased transit time of sound across the array, leading to snapshotdeficient processing [9]. In this research, a 30-element VLA is used to process the received signals from the target in the presence of ambient noise. The effect of motion on the eigen-structure of the sample covariance matrix is also explored.

This research investigates the vertical arrival structure of shipping noise and the passive detection of the target in the presence of ambient noise. The rest of this thesis is organized as follows. 
Chapter 1 gives the background on passive sonar and reviews the underwater acoustic wave propagation model.

Chapter 2 describes simulation models including the HITS database, Ambient Noise Directional Estimation System (ANDES) noise model, source depth model, and Ocean Acoustics and Seismic Exploration Synthesis (OASES) wind noise model. Together with the KRAKEN propagation model introduced in Chapter 1, these models were all used to simulate the ambient noise in the deep ocean environment. These models are applied to predict the directionality and array responses.

Chapter 3 reviews the beamforming principles, including conventional beamforming and adaptive beamforming techniques. The source motion analysis of nearby interferers is also explored.

Chapter 4 presents Monte Carlo simulations of the surface shipping noise. Results are compared with published experimental measurements conducted by Gaul et al. in [1]. The ambient noise levels are shown to be successfully modeled in complicated deep water environments as demonstrated by agreement with [1]. A VLA is used to investigate target detection performance in the presence of shipping noise and wind noise using passive sonar. The results from different beamforming methods show the effect of isolating the target from the ambient noise. Eigenvalue spectra analysis is utilized to investigate the nearby 
interference motion effects and results show motion effects can be minimized using short observation time.

Finally, Chapter 5 summarizes this research and suggests potential areas of future research.

\subsection{Sonar System}

SONAR (SOund Navigation And Ranging) is an underwater acoustic technique that uses acoustic signals to navigate, detect and localize targets [10]. The acronym SONAR was adopted in the 1940s. Attenuation of radio waves in water environment makes SONAR better suited to underwater remote sensing than RADAR (RAdio Detection And Ranging).

\subsubsection{Basic Concepts}

Sonar systems can be divided into two types: active and passive [10]. For ocean acoustics, in active sonar systems, sound waves (typically chirps or other broadband signals) are generated by a transmitter travel through the ocean to the target and the echoes are analyzed at the receiver. The time delay between the signal's departure and the reception of the echo is then used to determine the range of the target.

In comparison, passive sonar systems use hydrophones alone to listen to 
the sound generated by a self-emitting target. With a highly directional receiver, a passive sonar system can determine the signal arrival direction [11]. In contrast to active sonar where sound travels via two different wave propagation paths, passive sonar only consists of one-way transmission. Before the sound reaches the hydrophones, it may be reflected and scattered by the ocean media. Since World War II, military passive sonar systems have been developed toward lower frequencies in order to use the tonal or line components in the low-frequency submarine noise spectrum. Passive sonar arrays placed near the deep ocean bottom take advantage of the quiet environment and good propagation conditions for such low frequencies [10].

In complicated ocean environments, there are diverse sources of noise which can decrease the Signal-to-Noise Ratio (SNR) and degrade the performance of the sonar system. In this work, simulations of a passive sonar system are used to study the sound emitted from surface ships and characterize the noise structure in order to separate target from the ambient noise.

\subsubsection{The Sonar Equation}

The performance of a passive sonar system, can be described by the sonar equation (in $\mathrm{dB})[2]$ :

$$
S E=S L-T L-N L+D I-D T
$$


where

- SE (signal excess) - the value of that the actual received signal at the output of the beamformer exceeds the minimum signal for detection.

- SL (source level) - the value represents the loudness or amplitude of an acoustic source.

- TL (one-way transmission loss) - the value accounts for the energy lost due to the geometric spreading of the wave.

- NL (noise level) - the noise at a single hydrophone element.

- DI (directivity index) - the value represents the gain of the signal over the background noise, introduced by using an array.

- DT (detection threshold) - a parameter used to force a minimum level of the received signal in order to indicate a detection, and it is used to account for sonar operators training and alertness, among other factors.

\subsection{Underwater Acoustic Wave Propagation and Normal Modes}

The propagation of sound in the ocean can be interpreted and described mathematically by solutions of the wave equation using the appropriate boundary 
conditions and sediment parameters for a specific environment. The wave equation is presented as a partial differential equation, given by [10]

$$
\nabla^{2} p-\frac{1}{c^{2}(\vec{r})} \frac{\partial^{2} p}{\partial t^{2}}=0
$$

where $p$ is the acoustic pressure corresponding to the coordinates, $t$ is the time, $\vec{r}$ is the vector position of the point where the pressure is being measured, $c(\vec{r})$ is the sound speed profile as a function of the position $\vec{r}$, and $\nabla^{2}$ is Laplacian defined in some coordinate system according to the geometry of the waveguide.

There are two popular theoretical methods to solve the wave equation [10], which are ray theory and normal mode theory. Ray theory is a method normally used to model broadband acoustic propagation or at high frequencies. Normal mode model works in the frequency domain, and it separates the wave equation into its depth and range dependency. At each frequency, a number of modes (or solutions) to the wave equation can be computed, and the solution is the summation of all the modes at each particular frequency. Since the number of modes is proportional to the frequency, this model is computationally intensive at high frequencies. In this research, normal mode theory is applied, since the sources are narrowband and operate at frequencies lower than $300 \mathrm{~Hz}$. In order to solve equation (1.2), we proceed by adopting a cylindrical coordinate system with axial symmetry, which is the most appropriate model for an ocean 
acoustic channel. The solution of the wave equation provides the value of the acoustic pressure at any range and depth, and it can be written as:

$$
p(r, z)=\frac{i}{\rho \pi \sqrt{8 \pi r}} e^{-i \pi / 4} \sum_{m=1}^{M} \Psi_{m}\left(z_{s}\right) \Psi_{m}(z) \frac{e^{i k_{r m} r}}{\sqrt{k_{r m}}}
$$

where $r$ is the source-receiver range, $z$ is the depth of the receiver, $\rho$ is the density of the water, $\Psi_{m}(z)$ is the $m^{t h}$ mode function, $z_{s}$ is the depth of the source, and $k_{r m}$ is the horizontal wave number. In equation (1.3), $M$ is the total number of modes. Frequency dependence is included in the definition of wave number $k_{r m}=\omega / v_{p}$, where $v_{p}$ is the phase velocity.

From equation (1.3), the pressure as a function of the range $r$ and depth $z$ is a summation of $M$ different modes, each of which is a solution to the wave equation, with amplitude and phase given by $\Psi_{m}\left(z_{s}\right) \Psi_{m}(z) / \sqrt{k_{r m}}$ and $k_{r m} r$, respectively.

In this research, surface ships are located many water depths away from the receiver. The acoustic pressure $p(r, z)$ as a function of the position of interferers can therefore be computed using the normal mode expansion as in (1.3).

KRAKEN normal mode program [12] is used to solve for the horizontal wave number $k_{r m}$ and the $m^{\text {th }}$ mode function $\Psi_{m}$ by numerical methods. The basic process is shown in Figure 1.1. The inputs to the model are the sound speed profile (SSP), the characteristics of the seabed (density, sound speed, 
number of layers and sediment attenuation), and the depths of the source and receiver. The outputs of KRAKEN are $k_{r m}$ and $\Psi_{m}$, which are used in equation (1.3) to calculate the acoustic pressure due to the target source and interferers.

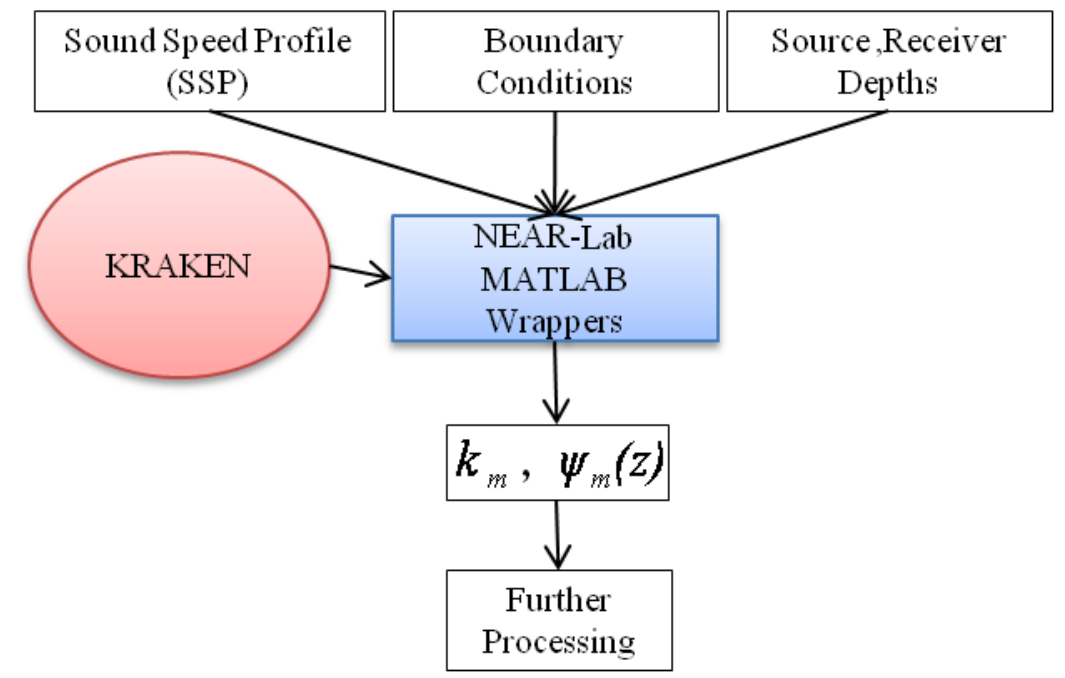

Figure 1.1: Flow chart of KRAKEN normal mode program.

Two examples of using KRAKEN to solve the wave equations are shown in Figure 1.2(a) and Figure 1.2(b). Both figures show transmission loss for the Munk profile, which is an idealized deep ocean sound speed profile shown in Figure 1.3. The source is placed at the depth of $50 \mathrm{~m}$ and the frequency is $50 \mathrm{~Hz}$. In Figure 1.2(a), the output includes 63 modes which are the waterborne modes only, while in Figure 1.2(b), the output includes 102 modes which are a combination of waterborne modes and bottom bounce modes. The sound propagation and interference patterns can be seen in these figures. In Figure 1.2(a), the most distinctive feature is the presence of the convergence 
zones, approximately every $65 \mathrm{~km}$ in range from the source. This convergence (or focusing) of energy is a common phenomena when the source is located near the surface and the sound speed profile has a shape as shown in Figure 1.3. In this case, the energy from the source propagates to the deep water due to the depth dependent decrease in the sound speed profile. At $1300 \mathrm{~m}$ depth, the sound speed increases again, causing an upward refraction of the energy which results in strong focusing at periodic intervals at the surface. Figure 1.2(b) shows also some of the modes that interact with the seabed at ranges of $18 \mathrm{~km}$ and $50 \mathrm{~km}$. Interference patterns can be observed when the bottom-reflected modes interact with the waterborne modes at short ranges (for example, at range $22 \mathrm{~km}$ and depth $4 \mathrm{~km}$ ). There is a strong attenuation associated with the bottom-reflected modes, because only a fraction of the energy is reflected back to the water column. Therefore, at long ranges waterborne modes are dominant. 


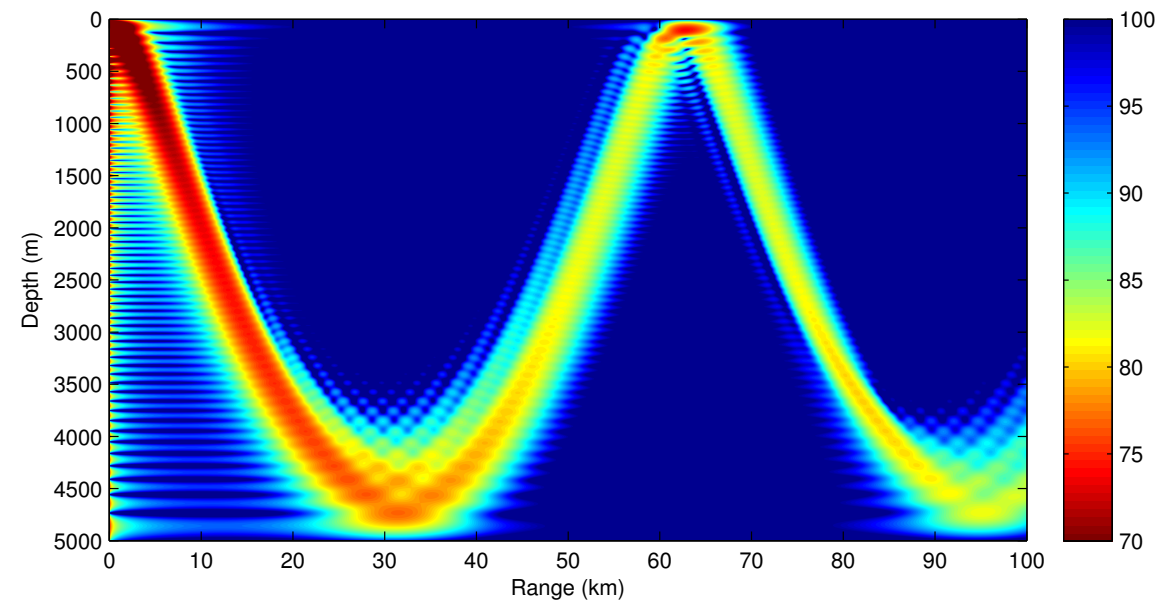

(a)

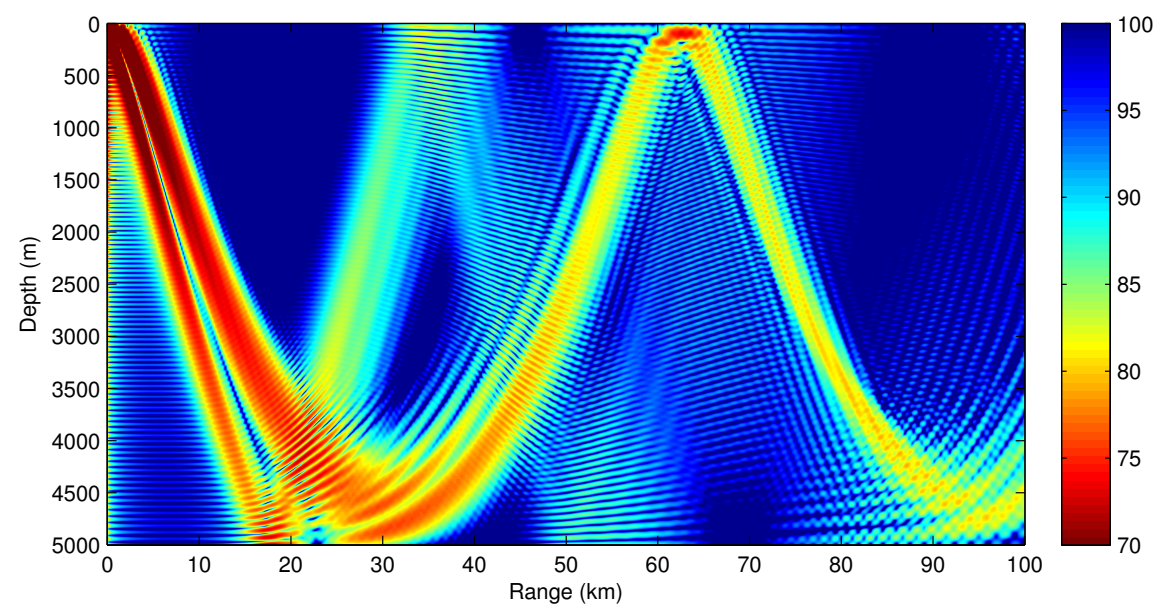

(b)

Figure 1.2: Transmission loss for the Munk profile with a source depth and frequency of $100 \mathrm{~m}$ and $50 \mathrm{~Hz}$, respectively, simulated by KRAKEN normal mode program including (a) 63 modes (waterborne modes only), and (b) 102 modes (waterborne modes and bottom bounce modes). 


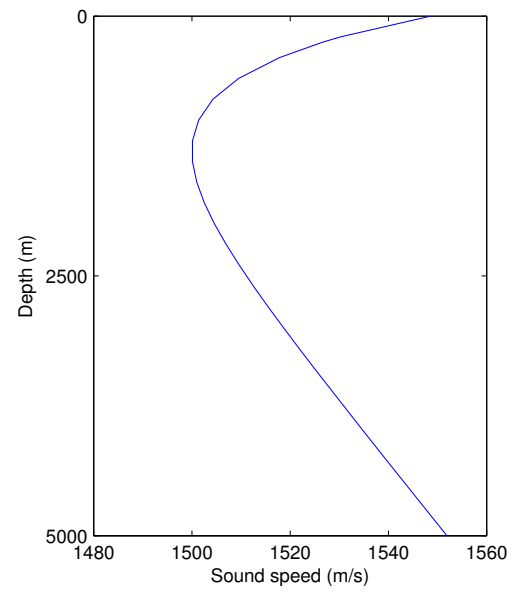

Figure 1.3: The Munk sound-speed profile. 


\section{Chapter 2}

\section{Signal and Noise Models}

In this chapter the models used to represent the signal received on the hydrophone are introduced. The HITS database is used to estimate the expected number of ships in a particular area. A Monte Carlo simulation based on this database is applied to examine the surface shipping noise. Source levels and source depths of the ships are assigned in agreement with the published

measurements and results [13] [15]. KRAKEN propagation model and Ocean Acoustics and Seismic Exploration Synthesis (OASES) wind noise model are used to analyze the acoustic field from surface ships and wind.

\subsection{Historical Temporal Shipping (HITS) database}

In most ocean areas, the dominant cause of ambient noise over the frequency band between 5 to $300 \mathrm{~Hz}$ is sound radiated by vessels [13]. Noise from surface shipping typically raises background spectral noise levels by 20 to $30 \mathrm{~dB}$ or more. In some areas, surface shipping noise dominates the noise spectrum up to $1000 \mathrm{~Hz}$. The average ambient noise spectra has been described by Urick [10] and is shown in Figure 2.1. 


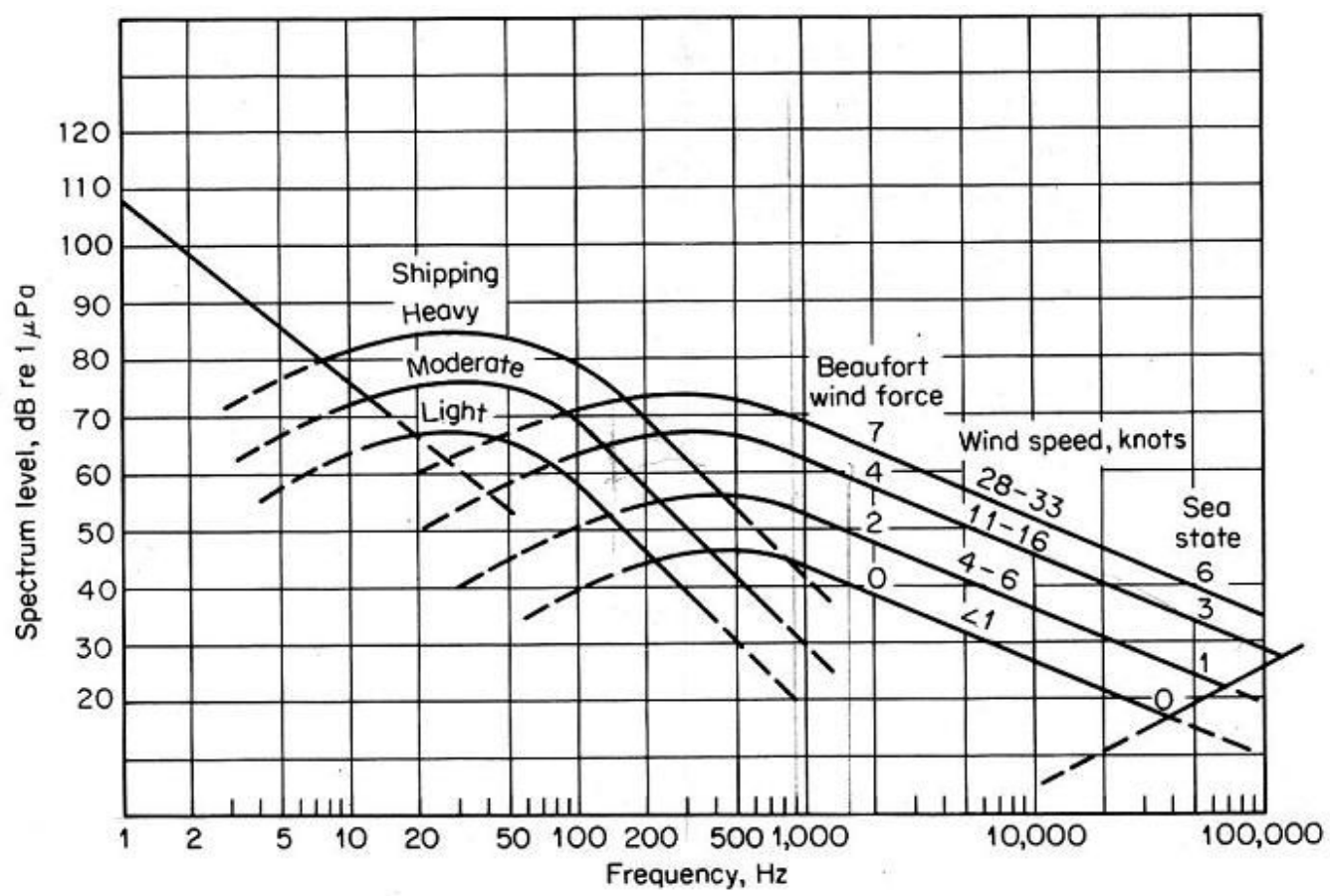

Figure 2.1: Average deep-water ambient noise spectra. Figure taken from Urick [10]

Knowledge of surface shipping traffic is important for predicting the directionality of ambient noise in the ocean. In order to predict the ambient noise radiated from surface ships, it is necessary to know (1) the spatial distribution of surface ships at a particular time, (2) how the spatial distribution changes in time, and (3) the sound radiation characteristics of the ships [13].

The HITS density database [13] provides monthly global shipping densities for vessels divided into the following five types: super tankers, large tankers, tankers, merchant vessels, and fishing vessels. The monthly density is given in units of ships per 1000 square nautical miles (ships/1000 $\mathrm{nm}^{2}$ ). This can be 
used to estimate the expected number of ships in a particular area. Resolution of the database is given by cells of $5 \mathrm{~nm} \times 5 \mathrm{~nm}$, and the ship count is calculated by the product of the density and the area of the cell. The ship count is the instantaneous number of ships instead of the number of ships that pass through the cell during a period of time. During a short snapshot length, the number of new ships entering in a cell and the number of ships leaving the cell are expected to be equal, which means the average ship count in a cell remains constant. However, the ship count in a cell may change over a long period of time, for instance, over several months. In this case, we can use Poisson distribution based on the database to generate 5000 realizations which represent 5000 moments in a month. For Poisson distribution, the mean in each cell is the ship number in each cell based on HITS database.

Statistics for fishing vessels were collected in 1999 by the Food and Agricultural Organization (FAO) and they do not follow distinct shipping lanes because their locations depend upon the distribution of marine species. Fishing vessels are active $31 \%$ of the time from October to March and $35 \%$ of the time from April to September. The data corresponding to all other ship types were collected based upon Lloyd's of London voyage data for 1998, and show lanes of heavy traffic between ports. Figure 2.2 shows the global merchant ship distribution in the month of December. Merchant shipping routes vary 
seasonally due to economic fluctuations [13].

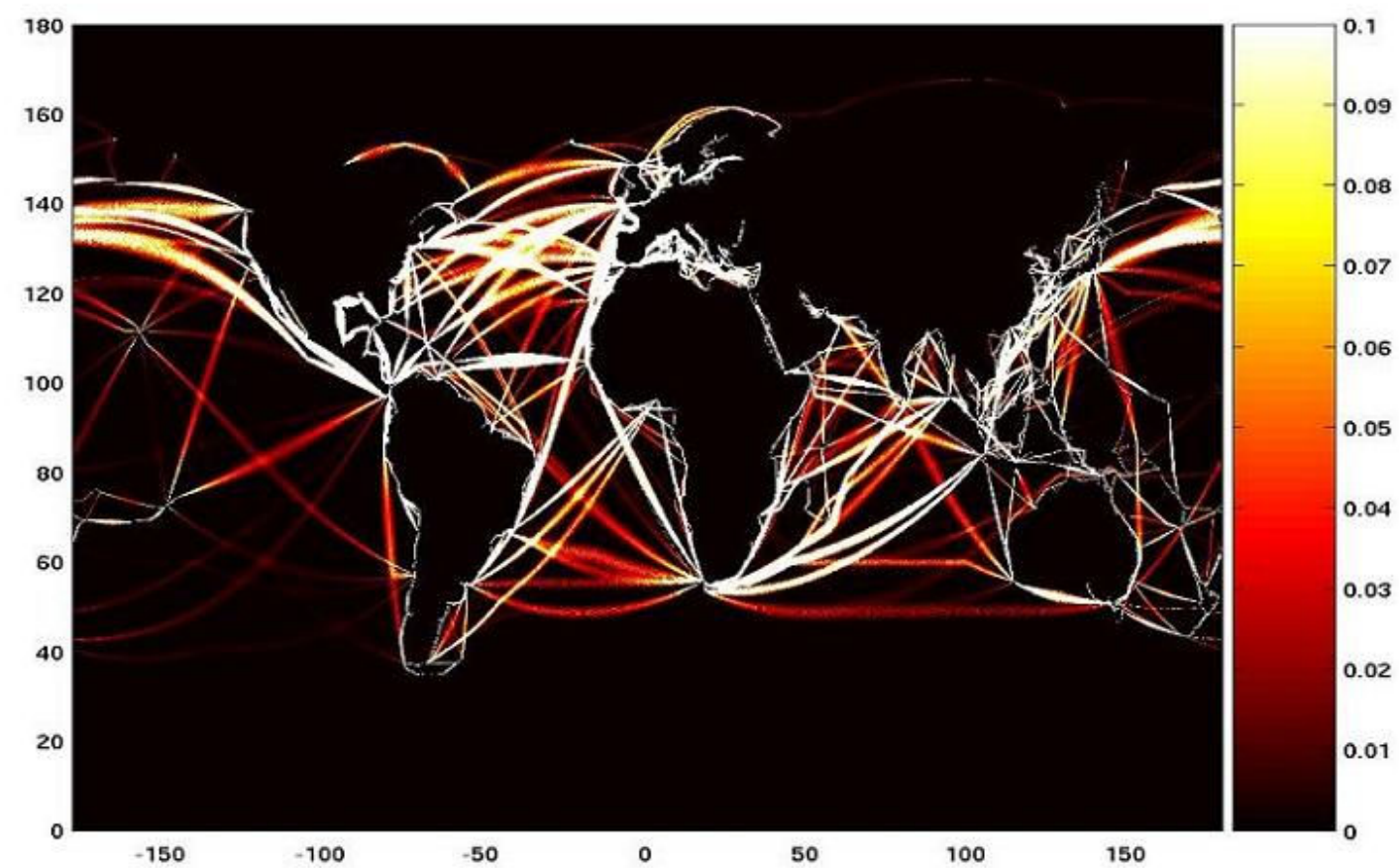

Figure 2.2: Global merchants distribution in December, 1998. Figure is generated based on HITS database.

In this research, surface ship noise is examined with a Monte Carlo simulation based on statistics derived from the HITS database. Details of this process can be found in Section 4.1.

\subsection{Source Level and Source Depth}

The present operational capability to predict ambient noise levels is provided by the Ambient Noise Directional Estimation System (ANDES) [14] developed in the early 1980s. The ANDES model analyzes the propagation of sound 
from surface ships and wind. It uses data of both the ocean environment and surface shipping patterns over the frequency band of 20 to $1000 \mathrm{~Hz}$. It is applied to predict ambient noise levels and directionality. The source level spectra calculated by ANDES model which is shown in Figure 2.3, depend only on ship classifications, not on ship length or speed. In this research, the ANDES model is used to assign the source level spectra.

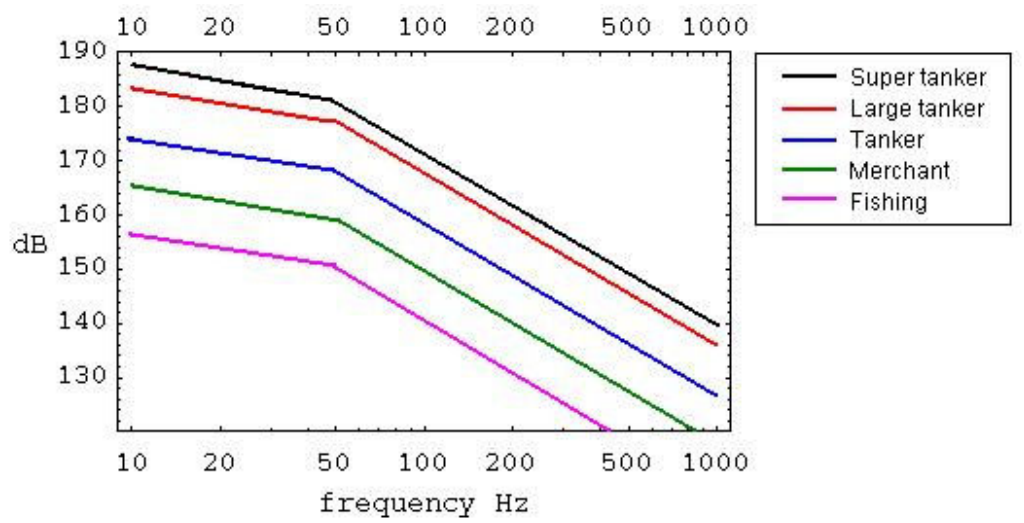

Figure 2.3: ANDES source level model [13]. The source level spectra calculated by ANDES model depend only on ship class, not on ship length or speed.

The source depth of each surface ship is assigned based on the ship draft, which is usually related to the ship length with a ratio of 1:20 [15]. In this research, the ship lengths are estimated as the average lengths based on ship classifications. Table 2.1 shows the source depths and source levels for different types of ships at the frequency of $50 \mathrm{~Hz}$ and $300 \mathrm{~Hz}$. 
Table 2.1: Source Depth (SD) and ANDES Source Level Model for Different Ship Types

\begin{tabular}{|c|c|c|c|}
\hline Ship Type & \multirow{2}{*}{ SD $(\mathrm{m})$} & \multicolumn{2}{|c|}{ ANDES Source Level Model } \\
\cline { 3 - 4 } & & $50 \mathrm{~Hz}(\mathrm{~dB})$ & $300 \mathrm{~Hz}(\mathrm{~dB})$ \\
\hline Super Tanker & 20 & 181.0 & 156.4 \\
\hline Large Tanker & 13 & 177.0 & 152.4 \\
\hline Tanker & 3 & 168.0 & 143.4 \\
\hline Merchant & 8 & 159.0 & 134.4 \\
\hline Fishing & 1.7 & 150.0 & 125.4 \\
\hline
\end{tabular}

\subsection{Wind Noise Model}

Wind noise is caused by wind turbulence near the surface of the sea. It is one of the primary sources of noise that affects sonar. Wind generates mechanical waves of different wavelengths which travel along the surface at different speeds. Waves with very long wavelengths can travel at speeds greater than the speed of sound in the water. In theory, the wind waves can radiate pressure waves into the depths of the sea. This mechanism is used to explain the principal characteristics of wind noise, such as its level and its variation with wind speed and frequency [10].

In this research, Ocean Acoustics and Seismic Exploration Synthesis (OASES) wind noise model is utilized. The OASES-OASN module [16] gives the seismoacoustic field on arbitrary three-dimensional array of hydrophones and geophones in the presence of surface noise sources and discrete signal sources. This module uses the wave-number integration method to model the propagation of 
surface-generated ambient noise and provides simulated array responses. The input of the frequency dependent noise level at different wind speed is obtained from the Wenz's curve [10]. The wind noise source level depend on the wind speed and frequency. Table 2.2 shows the relation between sea state and source strength and wind speed at the frequency of $50 \mathrm{~Hz}$. In this research, sea state is defined as the general condition of the free surface on a large body of water, with respect to wind waves, at a certain location and moment. The number of sea state adopts the "wind sea" definition of the Douglas Sea Scale.

Table 2.2: Sea State v.s. Source Strength and Wind Speed at $50 \mathrm{~Hz}$

\begin{tabular}{|c|c|c|}
\hline Sea State & Source Strength $(\mathrm{dB})$ & Wind Speed (knots) \\
\hline 1 & 45 & $4-6$ \\
\hline 3 & 59 & $11-16$ \\
\hline 6 & 68 & $28-33$ \\
\hline
\end{tabular}




\section{Chapter 3}

\section{Detection Performance}

Ocean acoustics often involves measuring and detecting a signal propagating in the ocean. A beamformer is used to estimate the narrowband signal arriving from a desired direction in the presence of noise and interferers with an array of sensors (receivers). The array collects spatial samples of propagating wave fields which are processed by the beamformer.

This chapter details the detection algorithm, which consists of beamforming techniques. Section 3.1 briefly introduces the array gain. Section 3.2 introduces the concepts of conventional beamforming and adaptive beamforming techniques, which will be used to detect the target in the presence of shipping noise and wind noise. Section 3.3 discusses how the motion of nearby interferers affects the performance of the algorithm.

\subsection{Array Gain}

An array of hydrophones is an acoustic antenna which can be used to amplify a signal coming from a specific direction. This has the effect of enhancing the SNR of sound passing the array at a certain angle relative to sound coming from other angles. Note that a single isotropic hydrophone records sounds at 
all directions with equal amplification. The degree to which SNR is enhanced by the array is called array gain (AG). It is defined in decibels as [10]:

$$
A G=10 \log \frac{(S / N)_{\mathrm{N}}}{(S / N)_{\mathrm{e}}}
$$

where $(S / N)_{\mathrm{N}}$ is the SNR at the N-element array terminals, and the $(S / N)_{\mathrm{e}}$ is the SNR at a single element of the array. Note that in this situation, the SNR over all the elements of the array is assumed to be the same for simplicity. The Directivity Index (DI) could be used to represent AG, when the signal is a plane wave and noise is isotropic. For an ideal array which is a linear array of $N$ hydrophones with spacing of half wavelength, in presence of isotropic noise, the DI is simplified as [2]

$$
D I=10 \log N
$$

\subsection{Beamforming Techniques}

The term "beamforming" originated from the fact that early spatial filters were designed to form pencil beams to receive a signal radiating from a specific location and attenuate signal from other locations [17]. This is useful in an environment with the presence of interference signals, because the spatial separation can be utilized to isolate signals of interest from interferers, since the desired and interfering signals usually are from different spatial locations. 
Fig 3.1 shows a narrowband beamformer, which processes the signal by sampling the propagating wave field in space.

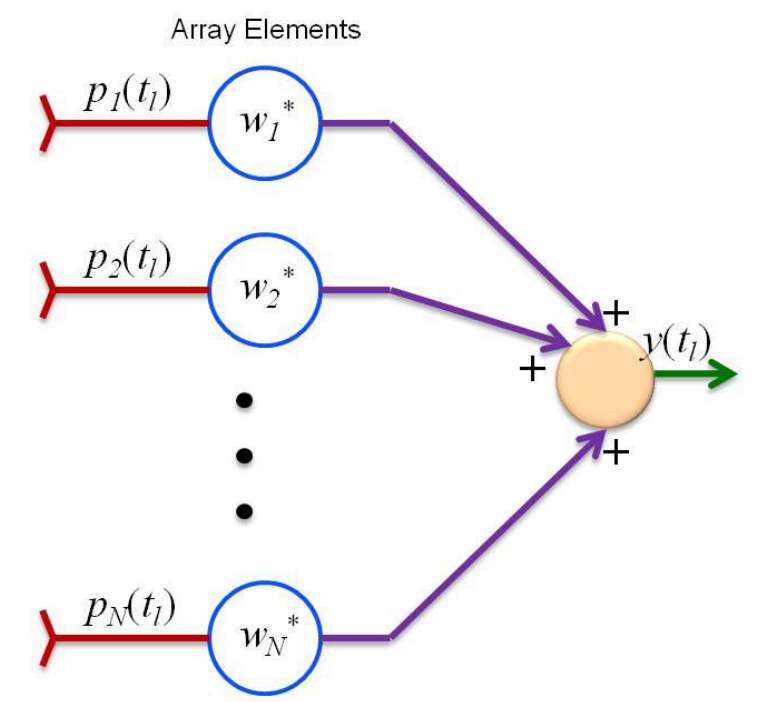

Figure 3.1: A narrowband beamformer forms a linear combination of the sensor outputs [17]. The outputs of sensors are each multiplied by a complex weight then summed.

At time $t_{l}$, the output $y\left(t_{l}\right)$ is given by [17]:

$$
y\left(t_{l}\right)=\sum_{n=1}^{N} w_{n}^{*} p_{n}\left(t_{l}\right),
$$

where $*$ represents complex conjugate, $N$ is the number of elements, $p_{n}\left(t_{l}\right)$ is the complex acoustic pressure of the $n^{\text {th }}$ element and $w_{n}$ is a complex weight factor used to steer the array in different directions. Therefore, $w_{n}$ can be thought as a spatial match filter with the angle of arrival of the assumed incident pressure as a parameter. This processing technique replaces the need 
of mechanically steering the array, which would be impractical in underwater acoustics.

For convenience, equation (3.3) can be written in vector notation as

$$
y\left(t_{l}\right)=\vec{w}^{H} \vec{p}\left(t_{l}\right)
$$

where $\vec{w}$ is the weight vector and $\vec{p}\left(t_{l}\right)$ is the $N \times 1$ data vector. The superscript $H$ represent the Hermitian transpose.

The quantity $\vec{w}$ is also known as a steering vector. There are a number of methods for deriving the individual vector elements. Two of these, known as conventional and adaptive, will be introduced in the next section.

\subsubsection{Conventional Beamforming}

From equation 1.2 , the position in three dimensional space was indicated as $\vec{r}$, independent of the coordinate system. In this research, normal modes are utilized and it is assumed that the environment is symmetric in the azimuth

coordinate. Then, a new vector notation $\Theta=(r, \theta, z)$ is introduced to denote position using range, elevation angle and depth. The array is an $N$-element array. The weight vector $\vec{w}$ in the conventional case is denoted as $\vec{v}\left(f_{0}, \Theta\right)$, which is the $N \times 1$ normalized weight vector called the replica vector or steering vector, and with appropriate normalization achieves unity gain in the direction 
of the target. The vector $\vec{v}$ is

$$
\vec{v}\left(f_{0}, \Theta\right)=\left[1 e^{i \sin \theta \cdot d \cdot 2 \pi f_{0} / c} \ldots e^{i(N-1) \sin \theta \cdot d \cdot 2 \pi f_{0} / c}\right]^{H}
$$

where $d$ is the spacing between elements of the array, and $c$ is the sound speed. The output of the array at frequency $f_{0}$ and direction $\Theta$ can be written as

$$
P\left(f_{0}, \Theta\right)=\vec{v}^{H}\left(f_{0}, \Theta\right) \hat{\mathbf{K}}\left(f_{0}, \Theta\right) \vec{v}\left(f_{0}, \Theta\right)
$$

where $\hat{\mathbf{K}}\left(f_{0}, \Theta\right)$ is the $N \times N$ sample covariance matrix. Here the time dependence is omitted by assuming that the sources are stationary. This assumption is accurate if the acoustic source represented in the covariance matrix $\hat{\mathbf{K}}$ does not change position during the sampling time in which snapshots are taken by the array for the estimation of $\hat{\mathbf{K}}$. This is often the case for slow vessels at long ranges from the array.

In this work, assuming sufficient snapshot support and uncorrelated sources, the covariance matrices can be written in their asymptotic form as

$$
\hat{\mathbf{K}}=\mathbf{K}_{\mathbf{T}}+\mathbf{K}_{\mathbf{s}}+\mathbf{K}_{\mathbf{w}}+\mathbf{K}_{\mathbf{n}}
$$

with 


$$
\begin{gathered}
\mathbf{K}_{\mathbf{T}}=\vec{p}_{\mathrm{T}} \vec{p}_{\mathrm{T}}^{H}, \\
\mathbf{K}_{\mathbf{s}}=\sum_{q=1}^{Q} \mathbf{K}_{\mathrm{s}, \mathbf{q}}=\sum_{q=1}^{Q} \vec{p}_{\mathrm{s}, q} \vec{p}_{\mathrm{s}, q}^{H}, \\
\mathbf{K}_{\mathbf{n}}=\sigma_{\mathrm{n}}^{2} \mathbf{I}
\end{gathered}
$$

where $\mathbf{K}_{\mathbf{T}}$ is the outer product of the input vector corresponding to the signal received from the target at the $N$ elements of the array. Similarly, $\mathbf{K}_{\mathrm{s}, \mathbf{q}}$ is the covariance corresponding to the $q^{\text {th }}$ surface shipping interferer, and there are Q interferers for each realization. The matrix $\mathbf{K}_{\mathbf{s}}$ is the summation of $\mathbf{K}_{\mathrm{s}, \mathbf{q}}$. The matrix $\mathbf{K}_{\mathbf{w}}$ is the wind noise calculated by OASES model and $\mathbf{K}_{\mathbf{n}}$ is the white noise matrix determined by white noise power $\sigma_{\mathrm{n}}^{2}$.

Conventional beamforming can perform very well in an interferer-free environment in which the channel properties are well-known. However, when there is uncertainty about the ocean parameters, adaptive beamforming outperforms conventional beamforming, even though it can suffer from excessive target signal loss and peak migration [18]. In addition, the beamwidth of conventional beamforming is very wide. If there are nearby interferers, it is difficult to isolate the target from nearby interferers with conventional beamformer. 


\subsubsection{Adaptive Beamforming}

For an adaptive beamformer, the output of an $N$-element array at frequency $f_{0}$ and direction $\Theta$ can be written as

$$
P\left(f_{0}, \Theta\right)=\vec{w}^{H}\left(f_{0}, \Theta\right) \hat{\mathbf{K}}\left(f_{0}, \Theta\right) \vec{w}\left(f_{0}, \Theta\right)
$$

where $\vec{w}\left(f_{0}, \Theta\right)$ is the $N \times 1$ weight vector related to the corresponding replica vector $\vec{v}\left(f_{0}, \Theta\right)$, and $\hat{\mathbf{K}}\left(f_{0}, \Theta\right)$ is the $N \times N$ sample covariance matrix. To suppress the interference sidelobes, the Minimum Variance Distortionless Response (MVDR) formulation is used [2].

The MVDR processor optimizes the response to a signal to be maximal in the look direction, while simultaneously attempts to reject signals and correlated noise from other directions [2]. The algorithm looks for a weight vector

$\vec{w}$ applied to the matrix $\hat{\mathbf{K}}$ which could minimize the output of the beamformer, except in the look direction where we expect the signal to pass through undistorted. Therefore, $\vec{w}$ is chosen to minimize the function [2]

$$
F=\vec{w}^{H} \hat{\mathbf{K}} \vec{w}+\lambda\left(\vec{w}^{H} \vec{v}-1\right)
$$

where $\lambda$ is the Lagrange multiplier [2]. Following the method of Lagrange 
multipliers, we obtain,

$$
2 \hat{\mathbf{K}} \vec{w}+\lambda \vec{v}=0,
$$

so that

$$
\vec{w}=-\frac{\lambda}{2} \hat{\mathbf{K}}^{-1} \vec{v},
$$

and

$$
\vec{w}^{H}=-\frac{\lambda}{2}\left(\hat{\mathbf{K}}^{-1} \vec{v}\right)^{H}=-\frac{\lambda}{2} \vec{v}^{H} \hat{\mathbf{K}}^{-1}
$$

Thus in the look direction, the constraint condition of unity gives

$$
\frac{\partial F}{\partial \lambda}=\vec{w}^{H} \vec{v}-1=0
$$

With equation (3.15) and equation (3.16), the Lagrange multiplier is

$$
\lambda=-2\left(\vec{v}^{H} \hat{\mathbf{K}}^{-1} \vec{v}\right)^{-1} .
$$

Combining equation (3.14) and equation (3.17), the MVDR weight vector in equation (3.18) is

$$
\vec{w}\left(f_{0}, \Theta\right)=\frac{\hat{\mathbf{K}}^{-1}\left(f_{0}, \Theta\right) \vec{v}\left(f_{0}, \Theta\right)}{\vec{v}^{H}\left(f_{0}, \Theta\right) \hat{\mathbf{K}}^{-1}\left(f_{0}, \Theta\right) \vec{v}\left(f_{0}, \Theta\right)},
$$

where $\hat{\mathbf{K}}$ is the sample covariance matrix given by equation (3.7). 
In order to illustrate the difference between conventional and adaptive techniques, Figure 3.2 shows the output of conventional beamforming and adaptive beamforming for three plane waves with incident angles of 0 degree, 30 degrees and 35 degrees, and source levels of $10 \mathrm{~dB}, 40 \mathrm{~dB}$ and $40 \mathrm{~dB}$, respectively. The array is 10 elements with spacing of half wavelength at $50 \mathrm{~Hz}$, and white noise is $10 \mathrm{~dB}$. This figure indicates that the adaptive beamforming with MVDR could resolve the two plane waves arriving at closed angles, while the conventional beamforming could not separate them. The weaker source can also be detected with adaptive beamforming.

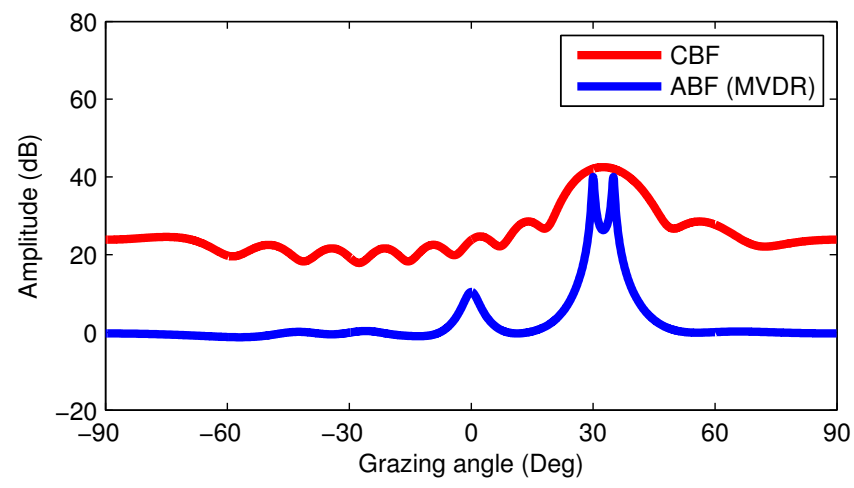

Figure 3.2: Comparison between conventional and adaptive beamforming at $50 \mathrm{~Hz}$. Two sources are at 30 degrees and 35 degrees, with $40 \mathrm{~dB}$ source level. One weak source is at 0 degree with $10 \mathrm{~dB}$ source level. The array is 10 elements with spacing of half wavelength, and white noise is $10 \mathrm{~dB}$. Adaptive beamforming with MVDR could resolve the two closed sources and detect the weak source, while conventional beamforming could not.

There are typically three sources of mismatch in adaptive beamforming 
processing, which are environmental, statistical and system [19]. Environmental mismatch is because of uncertainty in the propagation model, for instance, sound speed profile errors and bottom composition uncertainty. Statistical mismatch takes place when the number of degrees of freedom exceeds the number of available snapshots, which will be discussed in Section 3.3. System mismatch refers to errors in the receivers, for example, array tilt, hydrophone sensitivities and phase shifts.

The mismatch between the assumed signal and actual received signal in complicated underwater environments can lead to signal cancellation and prevent the detection of the actual signal [20]. This research implements diagonal loading of the covariance matrix using uniform loading levels to reduce signal self-nulling. The weight vector is given by equation (3.19) [7],

$$
\vec{w}\left(f_{0}, \Theta\right)=\frac{\left(\hat{\mathbf{K}}\left(f_{0}, \Theta\right)+\gamma I\right)^{-1} \vec{v}\left(f_{0}, \Theta\right)}{\vec{v}^{H}\left(f_{0}, \Theta\right)\left(\hat{\mathbf{K}}\left(f_{0}, \Theta\right)+\gamma I\right)^{-1} \vec{v}\left(f_{0}, \Theta\right)},
$$

where $\mathbf{I}$ is the identity matrix, and $\gamma$ is diagonal loading level. Diagonal loading level can be chosen to produce output between a fully adaptive beamformer $(\gamma=0)$ and the conventional beamformer $(\gamma=\infty)$. Therefore, the performance of diagonal loading can vary remarkably and it is important to find an optimal loading level. A loading level which is $5-10 \mathrm{~dB}$ above the noise level is usually a good selection [21]. 
Since the distant shipping noise arrives via shallow grazing angles, a VLA is used to produce vertical beams that isolate the surface interference from the nearby target. In Section 4.2, simulation results quantifying the beamformer output as a function of ocean environment, receiver aperture, and frequency are presented for both conventional and adaptive beamformers.

\subsection{Source Motion Analysis}

Application of adaptive beamforming techniques to target detection in deep ocean is complicated due to the effect of source motion. The interferer motion can increase the dimensionality of the interference subspace (a moving source might look like multiple sources) and reduce the effect of adaptive beamforming suppression. The dominant noise in cluttered sonar environments is shipping noise, which suggest a nonstationary environment at some time scale [9]. This time scale is determined by the speed of moving discrete interferer sources through a resolution cell. Sources with close ranges to the array moving at high speeds will cause large changes in the arrival angle received by the array, which suggests the use of short term stationarity assumptions for this case. This research also investigates the target detection performance in the presence of fast-moving strong interferers. Figure 3.3 shows the diagram of source motion. If the interferer moves a very short distance, during this period of time, the 
source may be still considered as one source. If the interferer moves a long distance due to rapid moving speed or longer duration of time, the source might look like multiple sources which lead to multiple peaks in the eigenvalue spectrum.

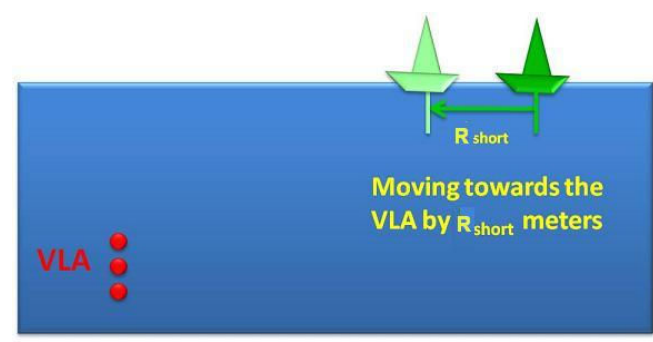

(a)

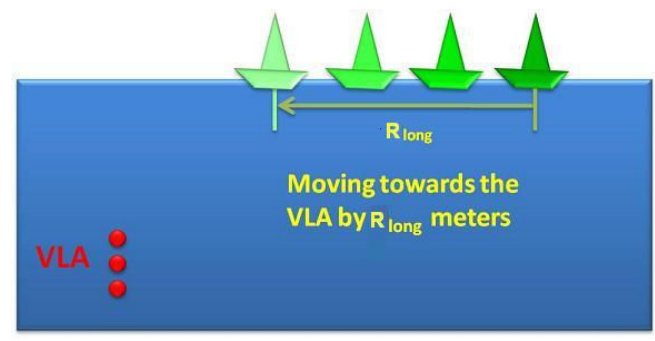

(b)

Figure 3.3: Source motion diagram. The interferer is moving towards the VLA. (a) The interferer moves a very short distance due to low moving speed or short time duration. In this case, the moving interferer can be still considered as one source. (b) The interferer moves in a long distance. In this case, the moving interferer might look like multiple sources.

Estimation of the sample covariance matrix in the presence of moving interferers over $L$ snapshots is given by,

$$
\hat{\mathbf{K}}_{\text {average }}=\frac{1}{L} \sum_{l=1}^{L} \vec{p}\left(t_{l}\right) \vec{p}^{H}\left(t_{l}\right)
$$

where $t_{l}$ represents the time of the $l^{t h}$ snapshot, and $\vec{p}\left(t_{l}\right)$ is the pressure at the receivers due to the target, ambient shipping noise and the moving interfering 
noise, given by

$$
\vec{p}\left(t_{l}\right)=\vec{p}_{\mathrm{T}}\left(t_{l}\right) e^{i \phi_{\mathrm{T}}\left(t_{l}\right)}+\sum_{q=1}^{Q} \vec{p}_{\mathrm{S}, q} e^{i \phi_{\mathrm{S}, q}}+\sum_{g=1}^{G} \vec{p}_{\mathrm{I}, g}\left(t_{l}\right) e^{i \phi_{\mathrm{I}, g}\left(t_{l}\right)} .
$$

For the $l^{\text {th }}$ snapshot, $\vec{p}_{\mathrm{T}}\left(t_{l}\right)$ is the pressure due to the target, $\vec{p}_{\mathrm{S}, q}$ is the pressure due to the $q^{\text {th }}$ stationary ship which can omit the time dependence, and $\vec{p}_{\mathrm{I}, g}\left(t_{l}\right)$ is the pressure due to the $g^{\text {th }}$ moving interferer. Additionally, $Q$ is the number of stationary ships and $G$ is the number of moving interferers. The random phases for target, $q^{\text {th }}$ stationary ship and $g^{\text {th }}$ moving interferer at $l^{\text {th }}$ snapshot are $\phi_{\mathrm{T}}\left(t_{l}\right), \phi_{\mathrm{S}, q}$ and $\phi_{\mathrm{I}, g}\left(t_{l}\right)$, respectively. These random phases are used to uncorrelate the signals from different sources.

Statistical mismatch refers to the need for covariance matrix, when the number of degrees of freedom usually exceeds the number of available snapshots. It is necessary to have the number of snapshots to exceed number of receivers to have full rank in the sample covariance matrix $\hat{\mathbf{K}}$. This suggests that the number of snapshots should be at least twice of the numbers of the elements of the array [21] for adaptive beamforming with MVDR. 


\section{Chapter 4}

\section{Simulation Results and Analysis}

In this chapter, simulation results for the vertical arrival structure of shipping noise and detection of target in ambient noise are presented. The details of the simulation process are presented in Section 4.1. Section 4.2 shows the results of exploring vertical noise structure and target detection performance using conventional and adaptive beamforming. The effect of moving interferers is also demonstrated.

\subsection{Simulation Process}

The simulations presented in this work explore the array performance under realistic conditions, and the results are compared the measurements presented by Gaul [1]. In this section, the environment and array parameters used to generate Monte Carlo realizations are described.

\subsubsection{Simulation Conditions}

In this research, the computed frequencies are $50 \mathrm{~Hz}$ and $300 \mathrm{~Hz}$. The purpose of selecting these frequencies is twofold: first, at $50 \mathrm{~Hz}$ and $300 \mathrm{~Hz}$ the ambient noise is dominated by shipping and wind noise, as shown in Figure 2.1. Second, 
because experimental measurements of ambient noise using deep arrays are available at these frequencies [1].

Location: The experimental measurements by Gaul [1] were taken in September 1975. Figure 4.1 shows the site of the experiments at Church Opal, located in NE Pacific with latitude and longitude of $27.7^{\circ} \mathrm{N}$ and $137.8^{\circ} \mathrm{W}$ respectively.

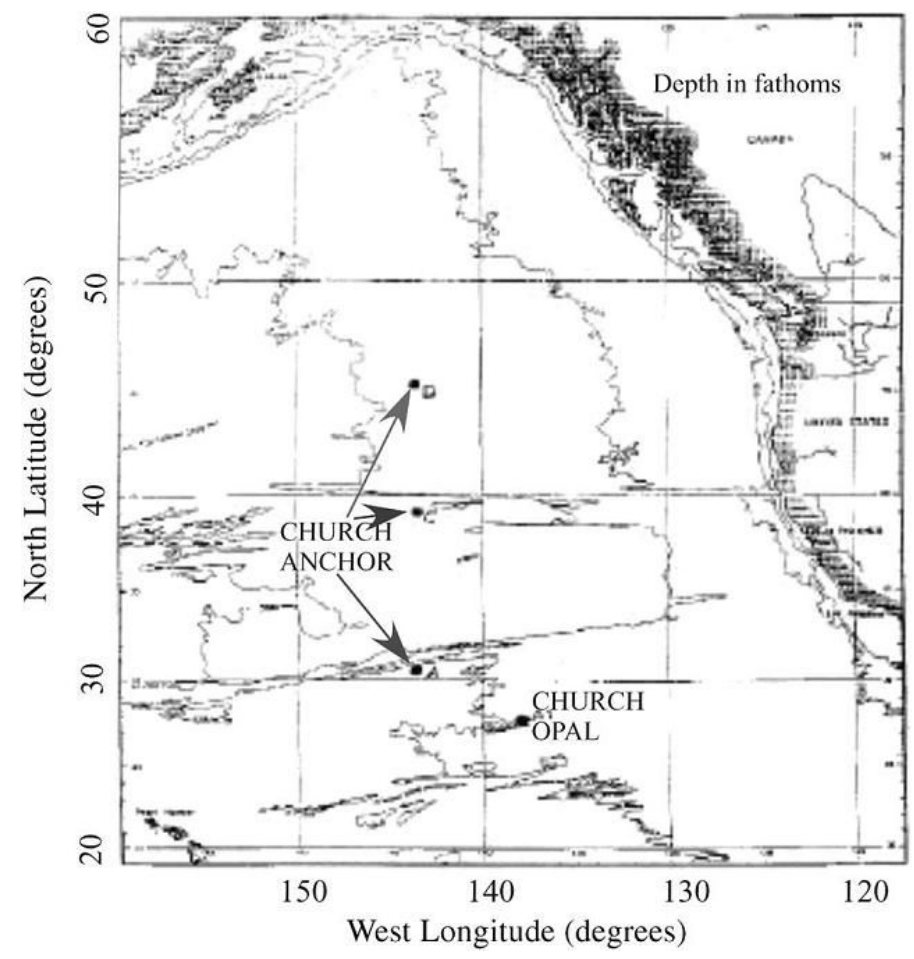

Figure 4.1: Locations of CHURCH OPAL measurement sites [1].

Ship Density: The shipping density data extracted from HITS database are at the same month and same location of Church Opal but extend the range from $22^{\circ} \mathrm{N}$ to $32^{\circ} \mathrm{N}$ and from $-142^{\circ} \mathrm{W}$ to $-127^{\circ} \mathrm{W}$. Monte Carlo simulations are used in this research and introduced in Section 4.1.2. 
One of the major purposes of the Church Opal experiment was to understand the nature of distant shipping noise in a deep water environment. In this research, distant ships are defined as ships with source to receiver range larger than $400 \mathrm{~km}$.

In addition, each ship is assigned with a source depth and a source level according to the ship classification in Table 2.1.

Environment parameters: Figure 4.2 gives the SSP at Church Opal [1]. The channel axis, the critical depth and the ocean bottom depth are $600 \mathrm{~m}, 4060$ $\mathrm{m}$ and $4880 \mathrm{~m}$ respectively.

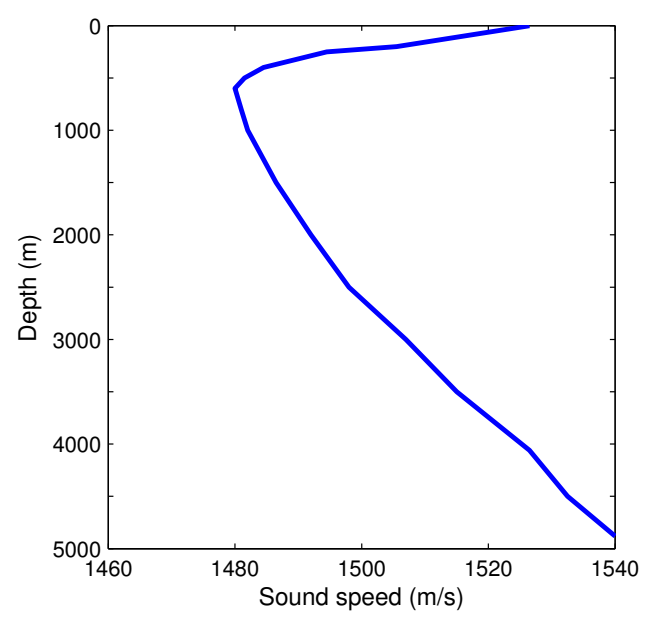

Figure 4.2: SSP at Church Opal in September, from Gaul et al [1].

Bottom parameters for the ocean channel model are chosen in agreement with the experimental observations from Hamilton [22] and shown in Table 4.1.

Wind Noise: For the simulations of vertical arrival structure from distant 
Table 4.1: Simulated Bottom Condition in Church Opal

\begin{tabular}{|c|c|c|c|}
\hline \multicolumn{2}{|c|}{ Frequency $(\mathrm{Hz})$} & 50 & 300 \\
\hline \multicolumn{2}{|c|}{ Bottom type } & Rigid & Rigid \\
\hline \multirow{5}{*}{ Sediment } & type & Silty clay & Silty clay \\
\hline & thickness $(\mathrm{m})$ & 100 & 100 \\
\hline & sound speed $(\mathrm{m} / \mathrm{s})$ & 1508 & 1508 \\
\hline & density $\left(\mathrm{g} / \mathrm{cm}^{3}\right)$ & 1.344 & 1.344 \\
\hline & $\alpha_{p}(\mathrm{~dB} / \mathrm{m})$ & 0.005 to 0.009 & 0.03 to 0.054 \\
\hline
\end{tabular}

shipping noise, wind noise corresponding to $5 \mathrm{kn}, 10 \mathrm{kn}$ and $15 \mathrm{kn}$ wind is considered. For the simulations of target detection performance, only wind noise corresponding to $5 \mathrm{kn}$ wind is considered.

VLA for Beamforming Simulations: Since the distant shipping noise arrives via shallow grazing angles, a VLA is used to produce vertical beams that isolate the surface interference from nearby targets. In order to explore the application of conventional and adaptive array processing techniques on target detection in a deep ocean environment, the VLA is an array of 30 elements with spacing between elements of $5 \mathrm{~m}$ for $50 \mathrm{~Hz}$ and $2 \mathrm{~m}$ for $300 \mathrm{~Hz}$, and it is located near the ocean bottom, as shown in Figure 4.3.

Simulated Target Conditions: The target is at $100 \mathrm{~m}$ depth and $5 \mathrm{~km}$ range. The classification of source level of the target is shown in Table 4.2. To explore the impact of target strength, two different sounds levels are computed, denoted as "average" and "noisy" targets. The values for these two levels are taken from Urick [10] and adjusted by approximately $10 \mathrm{~dB}$ (his values represent 


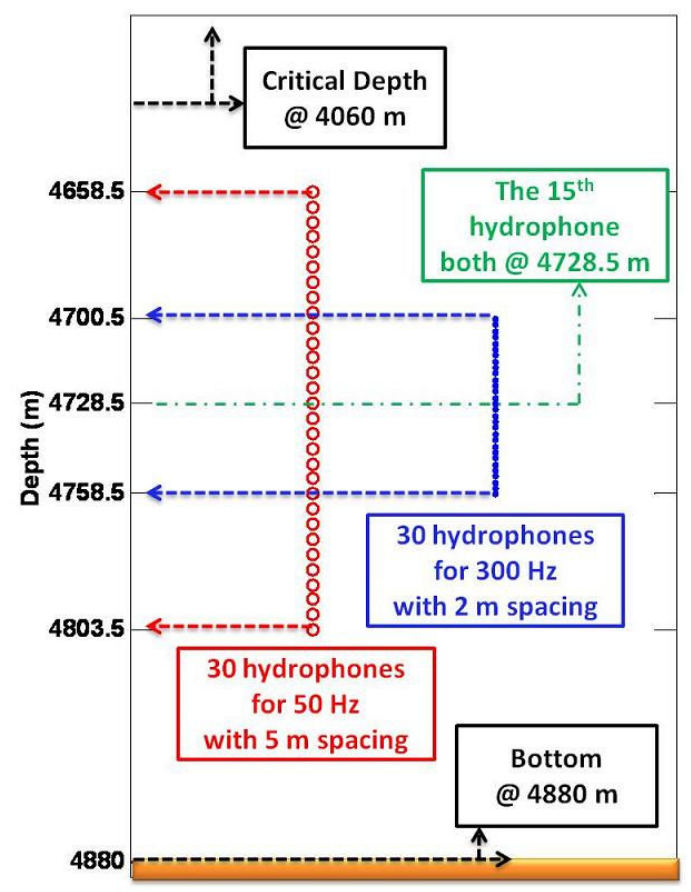

Figure 4.3: VLA for beamforming simulations.

broadband levels and are clearly historical, but they are used in an adjusted form here in the absence of more representative values).

Table 4.2: Source Level of the Target Classification

\begin{tabular}{|c|c|c|c|}
\hline \multicolumn{2}{|c|}{ Frequency } & $50 \mathrm{~Hz}$ & $300 \mathrm{~Hz}$ \\
\hline \multirow{3}{*}{ Source Level (dB) } & Quiet & 105 & 88 \\
\cline { 2 - 4 } & Average & 115 & 100 \\
\cline { 2 - 4 } & Noisy & 137 & 120 \\
\hline
\end{tabular}

\subsubsection{Monte Carlo Simulation Procedures}

Monte Carlo methods are a class of stochastic techniques based on using repeated random sampling and probability statistics to investigate problems. 
They consist of setting up a mathematical model for a system to be studied. In this research, the system is the environments described in the previous subsection. The input parameters such as number of ships and their positions relative to the array are modeled as random variables with an associated probability distribution. The Monte Carlo technique computes realizations of the received acoustic pressure by selecting the value of the input parameters from the assumed probability distributions. Therefore, an average over realizations gives the statistical mean of the received pressure.

In this work, Monte Carlo simulation is applied to analyze the vertical noise structure and investigate target detection performance. It includes 4 steps.

(1) Generate random ship distribution based on HITS database. Since the HITS database only provides the density in a whole month instead of each moment in the month, a Poisson distribution is used to generate 5000 realizations, representing 5000 moments in the month. The mean in each cell for the Poisson distribution is determined by the ship count calculated from monthly shipping density data.

(2) For each realization, calculate the pressure received by receivers from ships. For each realization, each ship is assigned with a source depth and a source level according to the ship classification as shown in Table 2.1. The complex pressure field radiated from each ship is computed using KRAKEN 
and the predicted values are summed coherently at each receiver location.

(3) Average over 5000 realizations to calculate vertical noise structure. Step

(1) to (3) provide the received level corresponding to the distant ships and wind for each realization. Additionally, wind noise is calculated by OASES model. Step (4) requires the average over 5000 realizations to present the vertical arrival structure from distant shipping noise to compare with the published experimental results $[1]$.

(4) Apply beamforming techniques on each realization and take an average over 5000 realizations. For each realization, beamforming techniques are applied to analyze the target detection performance in the presence of shipping noise and wind noise. White noise at $20 \mathrm{~dB}$ is added. The final beamforming output is the average over 5000 realizations of beamforming output.

\subsection{Simulation Results and Analysis}

Figure 4.4 shows the comparison between the statistics from HITS database and Monte Carlo realizations for large tankers in the month of September.

The shipping routes are clearly shown in Figure 4.4 which illustrate the lane-based representation in merchant distributions. The simulation results for fishing vessels, exhibit a uniform distribution because their locations depend on marine species. 


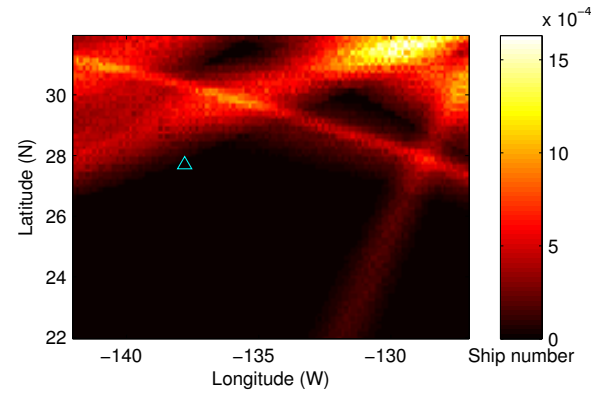

(a)

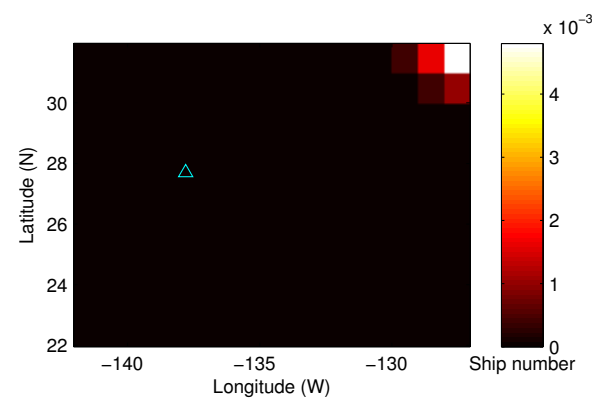

(c)

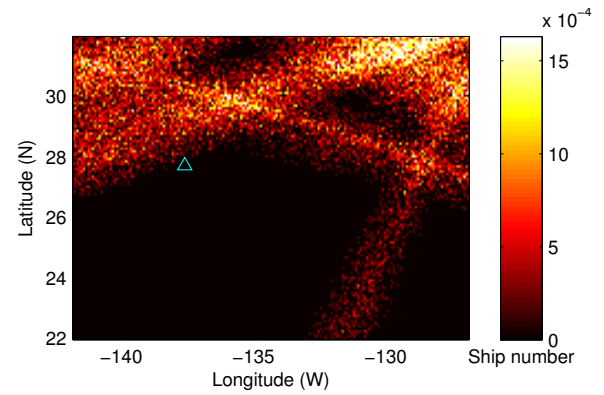

(b)

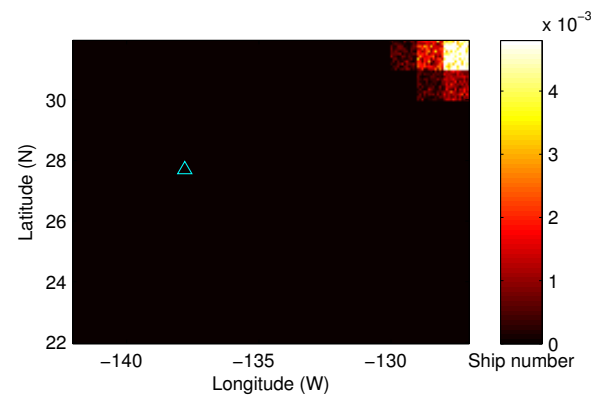

(d)

Figure 4.4: Comparison between the statistics from HITS database and Monte Carlo realizations in September: Statistics Data from HITS database for (a) large tankers and (c) fishing vessels; the average data of 5000 Monte Carlo realizations for (b) large tankers and (d) fishing vessels. The cyan triangle is the location of the VLA.

Figure 4.5 shows the pressure field of a super tanker at Church Opal using KRAKEN Propagation Model. The $50 \mathrm{~Hz}$ source corresponding to the super tanker is at a depth of $20 \mathrm{~m}$. The SSP is shown in Figure 4.2, and the boundary conditions are shown in Table 4.1. The water depth is $4880 \mathrm{~m}$ and the horizontal range is $400 \mathrm{~km}$. The simulation used 329 modes which includes the waterborne modes, bottom-bounce modes and leaky modes [2].

Figure 4.6 demonstrates simulated noise level at different sea state using the OASES wind noise model. The frequency is $50 \mathrm{~Hz}$. The source strength 


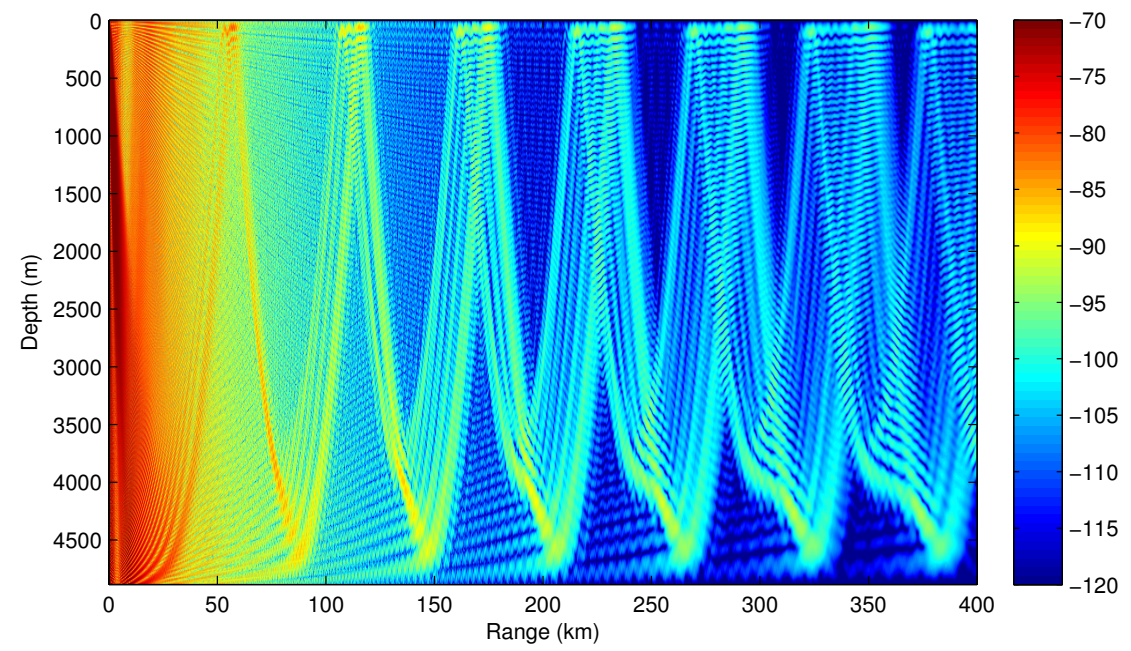

Figure 4.5: Transmission loss at $50 \mathrm{~Hz}$ for a super tanker calculated using KRAKEN propagation normal mode with 329 modes. The SSP and boundary conditions are given in Figure 4.2 and Table 4.1, respectively.

and wind speed for the different sea states are given in Table 2.2.

\subsubsection{Vertical Arrival Structure of Shipping Noise}

As introduced in Section 4.1, the simulation for interferers only includes distant shipping at a range larger than $400 \mathrm{~km}$. The received level is affected by the ship distribution on the ocean and the depth of the array. In order to analyze the effects of the receiver depth [23], Figure 4.7 shows the received level distribution from distant surface ships when the receiver is located at $50 \mathrm{~m}$ (near surface), $600 \mathrm{~m}$ (sound channel axis), $4060 \mathrm{~m}$ (critical depth), and $4850 \mathrm{~m}$ (near bottom), respectively. KRAKEN is used to compute the complex pressure field radiated from each interferer. Comparison of these four depths shows that the received 


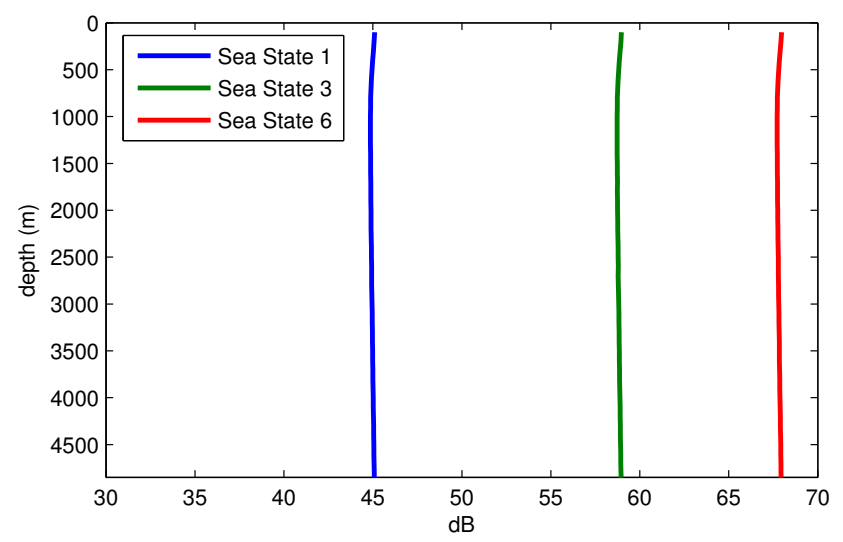

Figure 4.6: Simulated noise level at different sea states at $50 \mathrm{~Hz}$. The source strengths for these 3 sea states are $45 \mathrm{~dB}, 59 \mathrm{~dB}$, and $68 \mathrm{~dB}$ respectively. Wind speeds are 4-6 knots, 11-16 knots and 28-33 knots respectively.

levels for the VLA located near the surface and at critical depth have a similar pattern. The received level near the bottom is the smallest. This means that putting the array below the critical depth reduces the ambient noise, and might improve the target detection performance.

In the Church Opal experiment, receivers were positioned well below the critical depth in order to examine noise attenuation with depth and separate the wind and the distant shipping components of the ambient noise. From the measurement, it is apparent that there is a depth dependence of the noise at phones in and below the critical depth. This observation could be quantitatively explained by the effects of bathymetric shielding and bottom attenuation of noise from distant shipping [24]. Figure 4.8 shows the simulated ambient noise structure as a function of depth at $50 \mathrm{~Hz}$ and $300 \mathrm{~Hz}$. The wind noise corresponds 


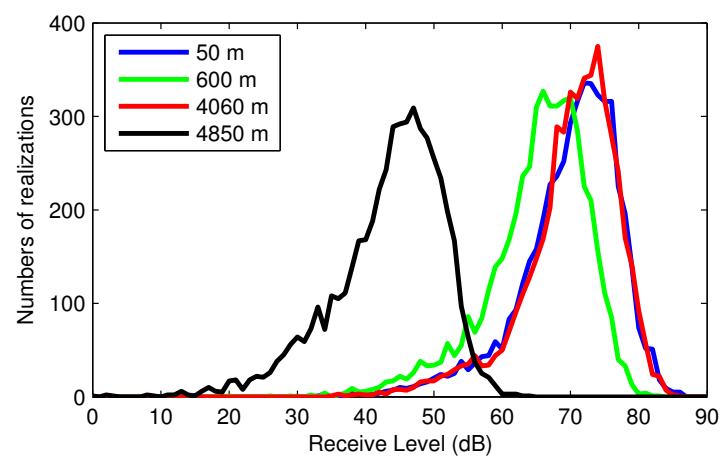

Figure 4.7: Distribution of the received levels from distant surface shipping for a receiver located at different depths at $50 \mathrm{~Hz}$ : The depths for the receiver are $50 \mathrm{~m}$ (near surface), $600 \mathrm{~m}$ (sound channel axis), $4060 \mathrm{~m}$ (critical depth), and $4850 \mathrm{~m}$ (near bottom), respectively. The received level near the bottom is the smallest. The received levels for VLA located near surface and located at critical depth have similar pattern.

to $5 \mathrm{kn}, 10 \mathrm{kn}$ and $15 \mathrm{kn}$ winds, respectively. The depth dependence of the simulated shipping noise is in agreement with the published experimental measurements [1] shown in Figure 4.9, which suggest that the noise from distant shipping may be significantly reduced when measured on receivers below the critical depth.

In these simulations, parameters for the ocean channel are chosen in agreement with the experimental observations, and sensitivity to the sediment thickness is explored. Figure 4.10 shows the dependency on bottom sediment layer parameters at $50 \mathrm{~Hz}$. The receiver is located $30 \mathrm{~m}$ above the ocean bottom, and the source depth is $10 \mathrm{~m}$. The source to receiver ranges are $200 \mathrm{~km}, 800$ km, $1500 \mathrm{~km}$ and $3000 \mathrm{~km}$, respectively. The bottom has a silty clay sediment. 


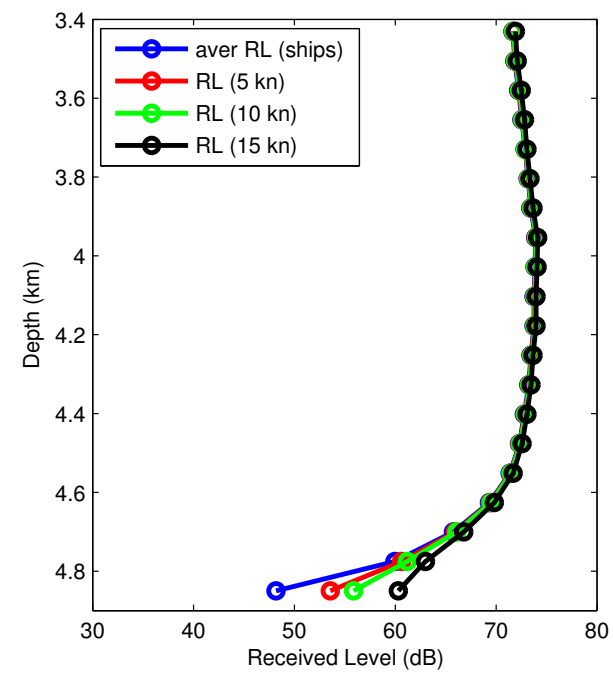

(a)

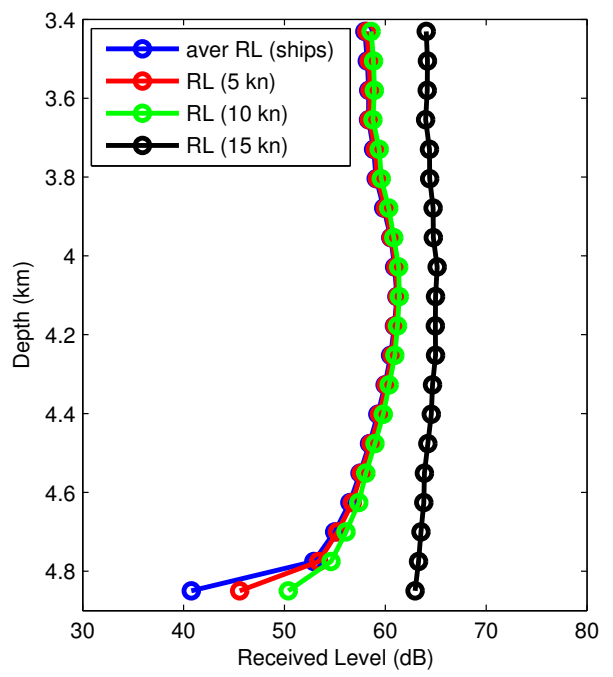

(b)

Figure 4.8: Simulated ambient noise levels as a function of depth at (a) 50 $\mathrm{Hz}$ and (b) $300 \mathrm{~Hz}$. Ambient noise includes surface distant shipping noise and wind noise. The wind noises correspond to $5 \mathrm{kn}, 10 \mathrm{kn}$ and $15 \mathrm{kn}$, respectively. The VLA is located near the bottom from $3430 \mathrm{~m}$ to $4850 \mathrm{~m}$.

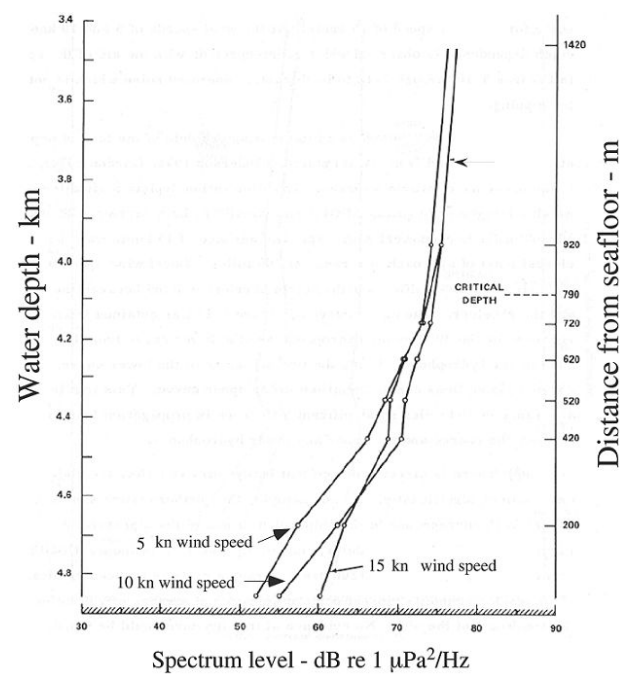

(a)

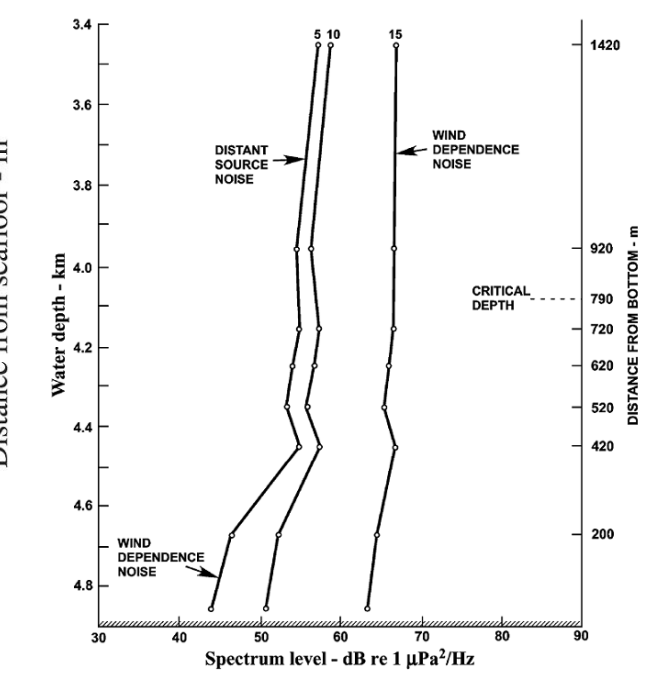

(b)

Figure 4.9: Measured ambient noise levels as a function of depth at (a) $50 \mathrm{~Hz}$ and (b) $300 \mathrm{~Hz}$. Figures are taken from Gaul [1]. 
The figure shows that a significant sediment thickness effect occurs when the source-receiver range is about $200 \mathrm{~km}$. This is because some of the energy penetrates into the sediment, and there is more transmission loss for a thicker layer. When the source-receiver range is much larger than $200 \mathrm{~km}$, the transmission loss is dominated by lower order modes, and the sediment thickness has less effect on the bottom transmission loss.

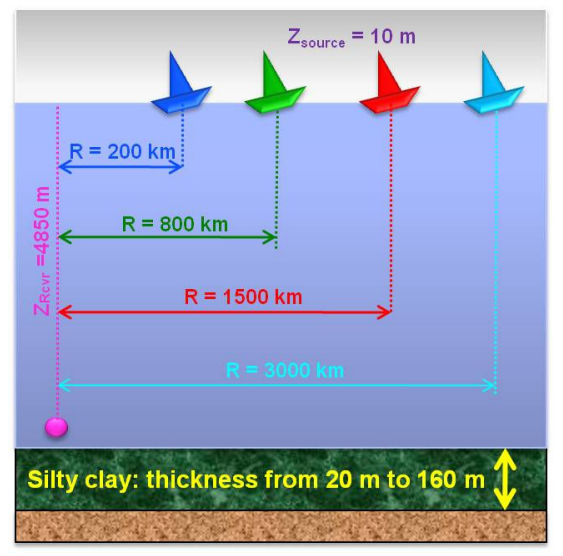

(a)

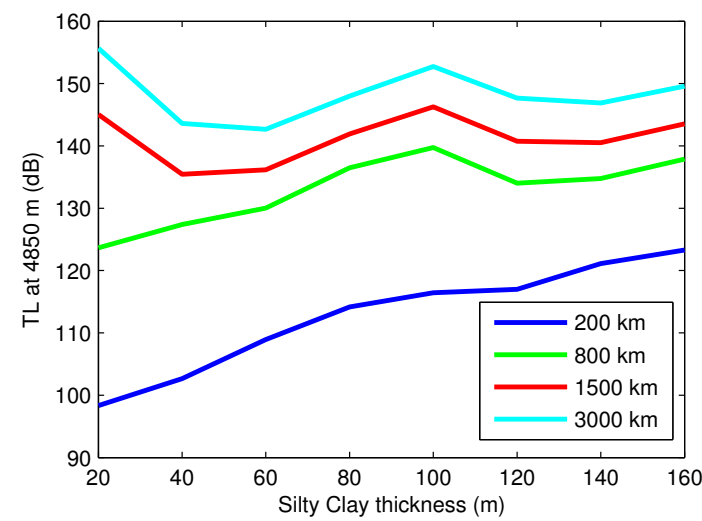

(b)

Figure 4.10: Computed bottom transmission loss as a function of sediment thickness. (a) Simulation geometry. The receiver is located at the depth of $4850 \mathrm{~m}, 30 \mathrm{~m}$ above the bottom. The source depth is $10 \mathrm{~m}$. The source to receiver ranges are $200 \mathrm{~km}, 800 \mathrm{~km}, 1500 \mathrm{~km}$ and $3000 \mathrm{~km}$, respectively. (b) Computed bottom transmission loss as a function of sediment thickness at 50 $\mathrm{Hz}$ for a layer of silty clay sediment. 


\subsubsection{Beamforming and Directionality for the VLA}

In this section, the target detection performance in the presence of distant shipping noise and wind noise with different beamforming techniques is presented. Figure 4.11 depicts the output of conventional beamforming with 45 dB Chebyshev taper at $50 \mathrm{~Hz}$, while Figure 4.12 shows similar results at 300 Hz. The target arrival angle is different from the distant shipping noise which is at 0 degrees and the wind noise which is between 60 and 90 degrees. The bottom bounce of the target is also detectable. The noisy target has better detection performance than the average one. At both 50 and $300 \mathrm{~Hz}$, the target arrival angle is approximately 45 degrees, which are shown in the red line, and the target bottom bounce is at approximately -45 degrees. For noisy target, conventional beamforming could detect the direct arrival angle, while the beamwidth is large. It is also interesting to note that the bottom bounce may prove a more robust detection path because it does not compete with the surface wind noise (as the direct target path does). However, the strength of the bottom bounce will depend on the bottom sediment properties.

Figure 4.13 shows the adaptive beamformer response without diagonal loading at $300 \mathrm{~Hz}$. The beamforming output has narrower beams for target and shipping noise. Figure 4.14 shows the adaptive beamformer response with 25 dB diagonal loading at $300 \mathrm{~Hz}$. 


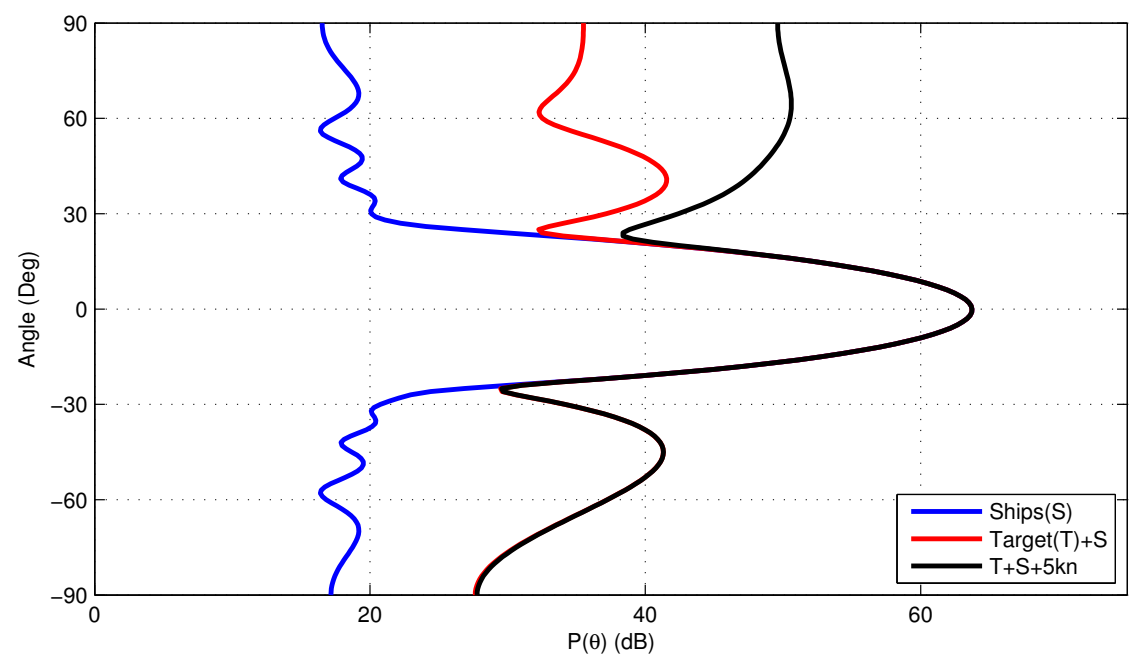

(a)

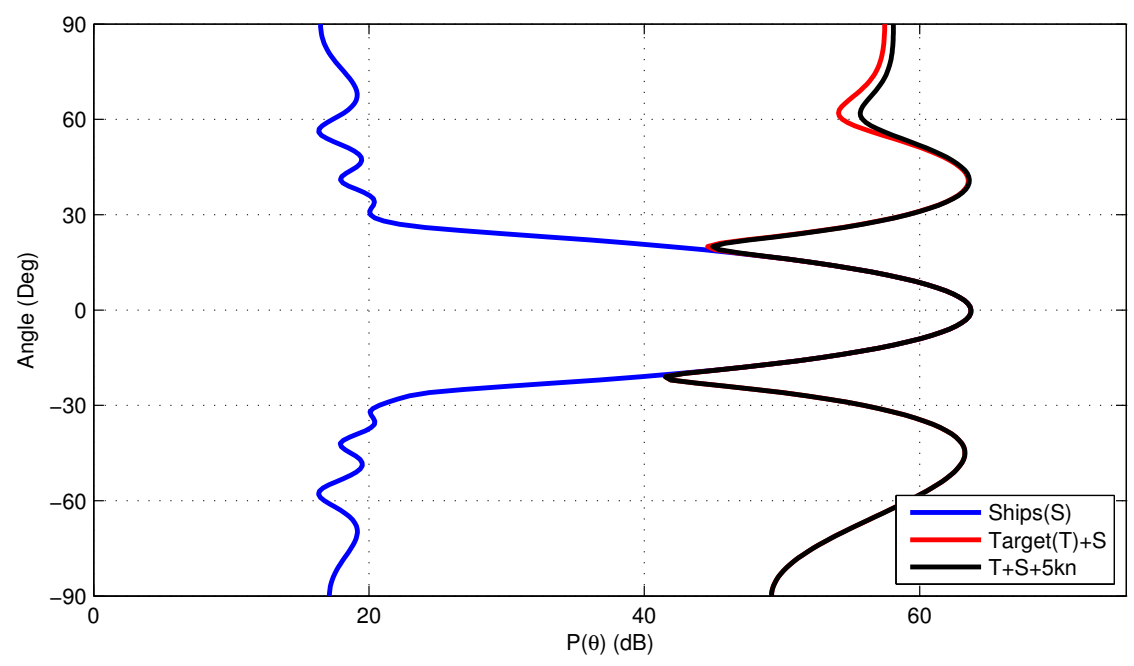

(b)

Figure 4.11: Conventional beamforming with $45 \mathrm{~dB}$ Chebyshev Taper at 50 $\mathrm{Hz}$. The target is at $5 \mathrm{~km}$ range, $100 \mathrm{~m}$ depth. Noise includes distant shipping noise, $5 \mathrm{kn}$ wind noise and $20 \mathrm{~dB}$ white noise. Target source levels are (a) 115 $\mathrm{dB}$ (average source level) and (b) $137 \mathrm{~dB}$ (noisy source level). 


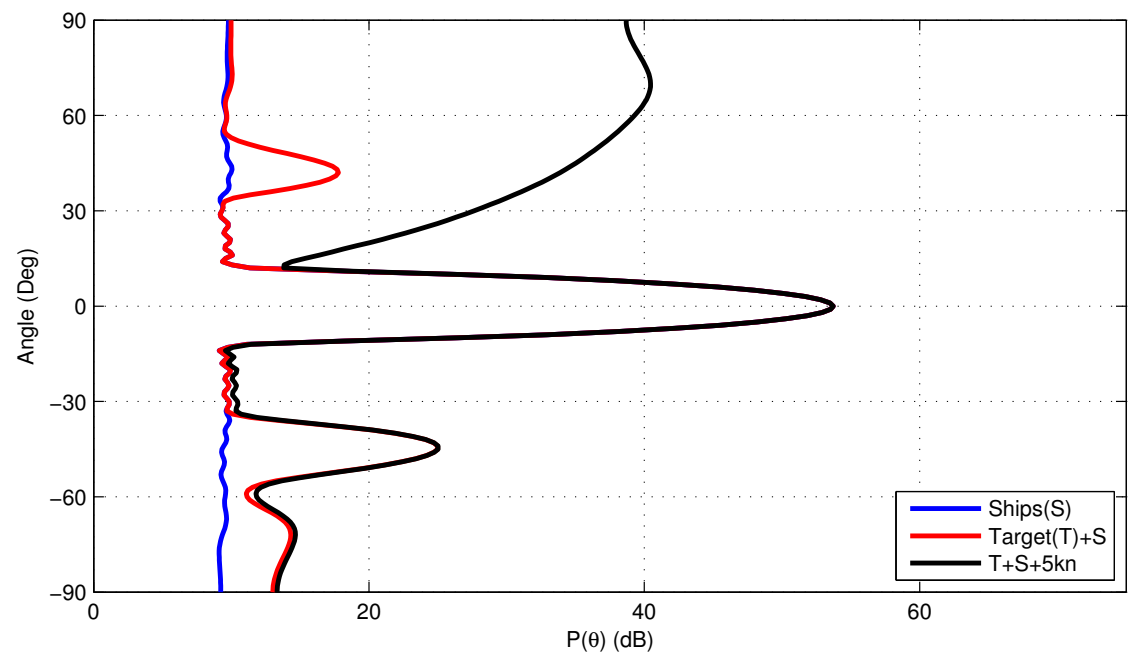

(a)

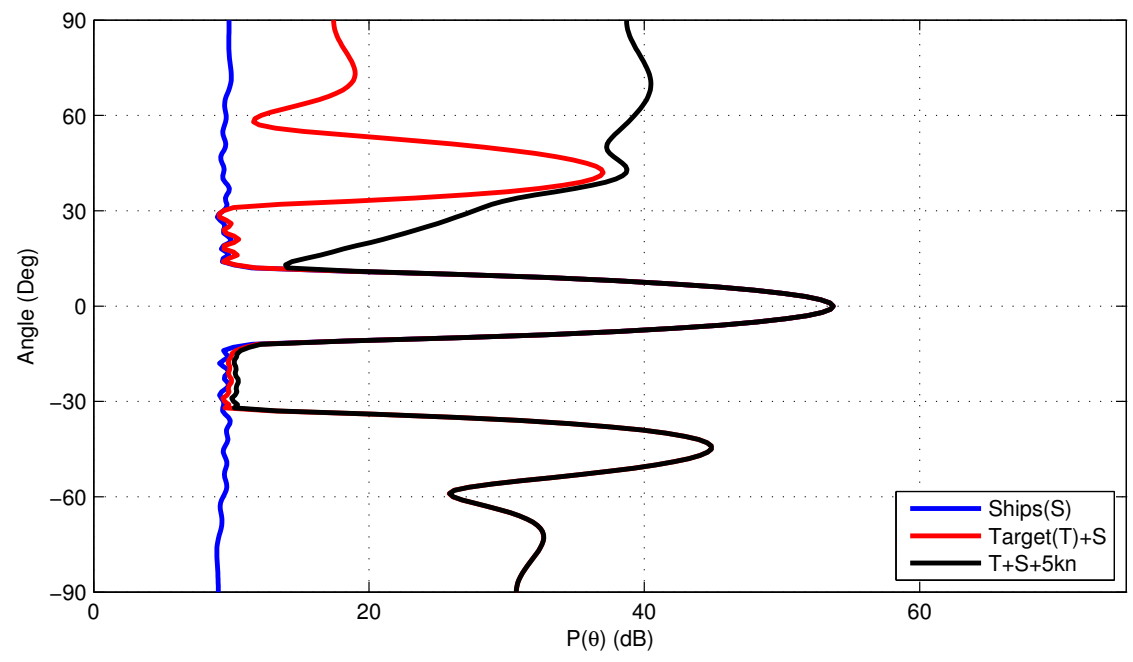

(b)

Figure 4.12: Same as Figure 4.11 with a higher frequency of $300 \mathrm{~Hz}$. Target source levels are (a) $100 \mathrm{~dB}$ (average source level), and (b) $120 \mathrm{~dB}$ (noisy source level). 


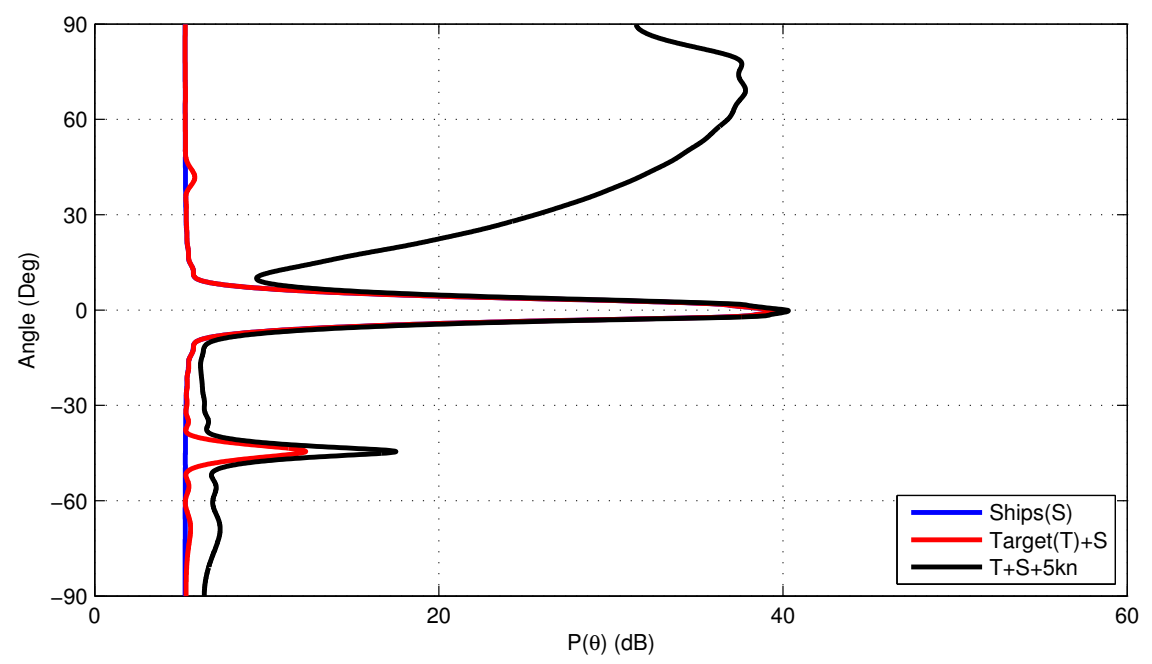

(a)

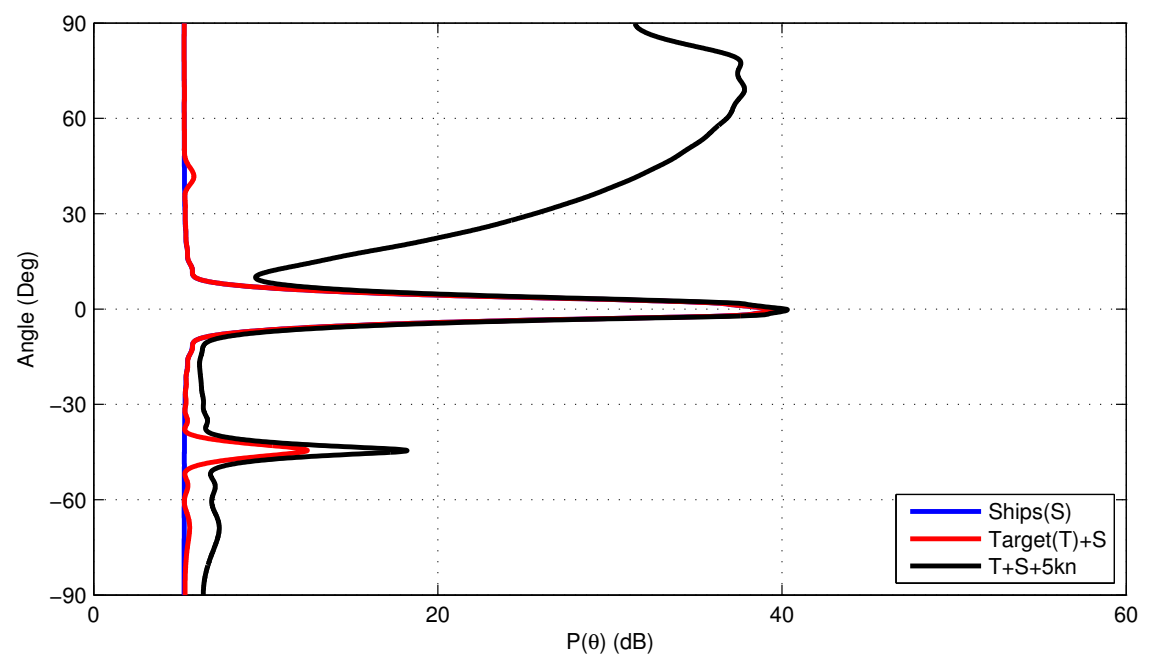

(b)

Figure 4.13: Adaptive beamforming without diagonal loading at $300 \mathrm{~Hz}$. The target is at $5 \mathrm{~km}$ range, $100 \mathrm{~m}$ depth. Noise includes distant shipping noise, $5 \mathrm{kn}$ wind noise and $20 \mathrm{~dB}$ white noise. Target source levels are (a) $100 \mathrm{~dB}$ (average source level), and (b) $120 \mathrm{~dB}$ (noisy source level). 


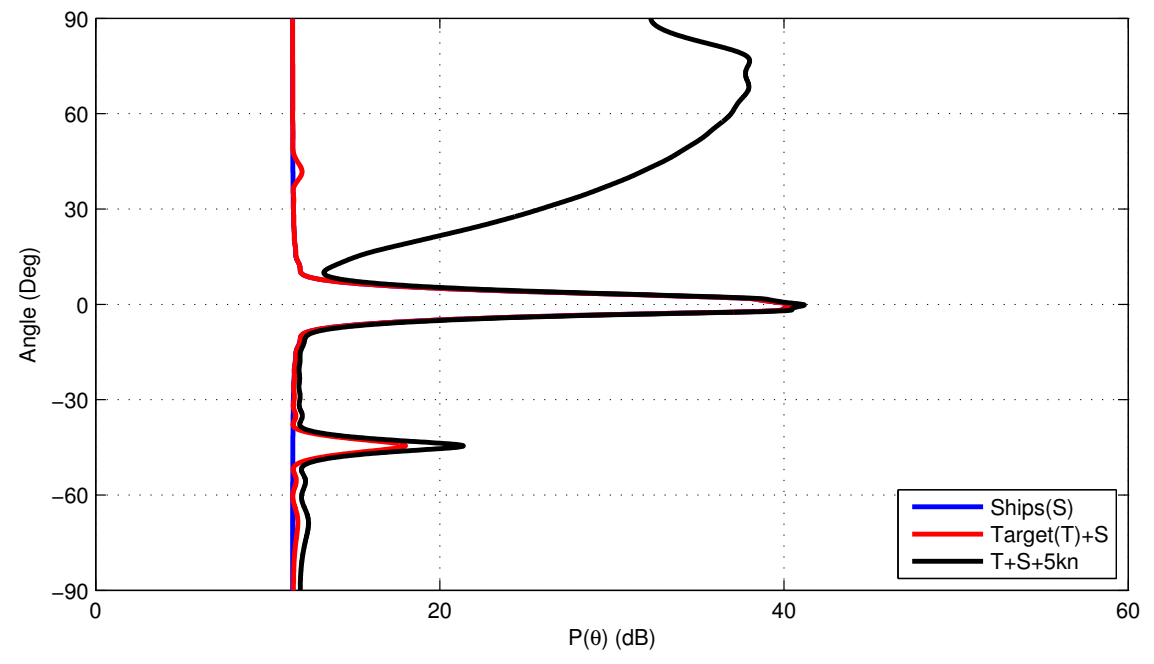

(a)

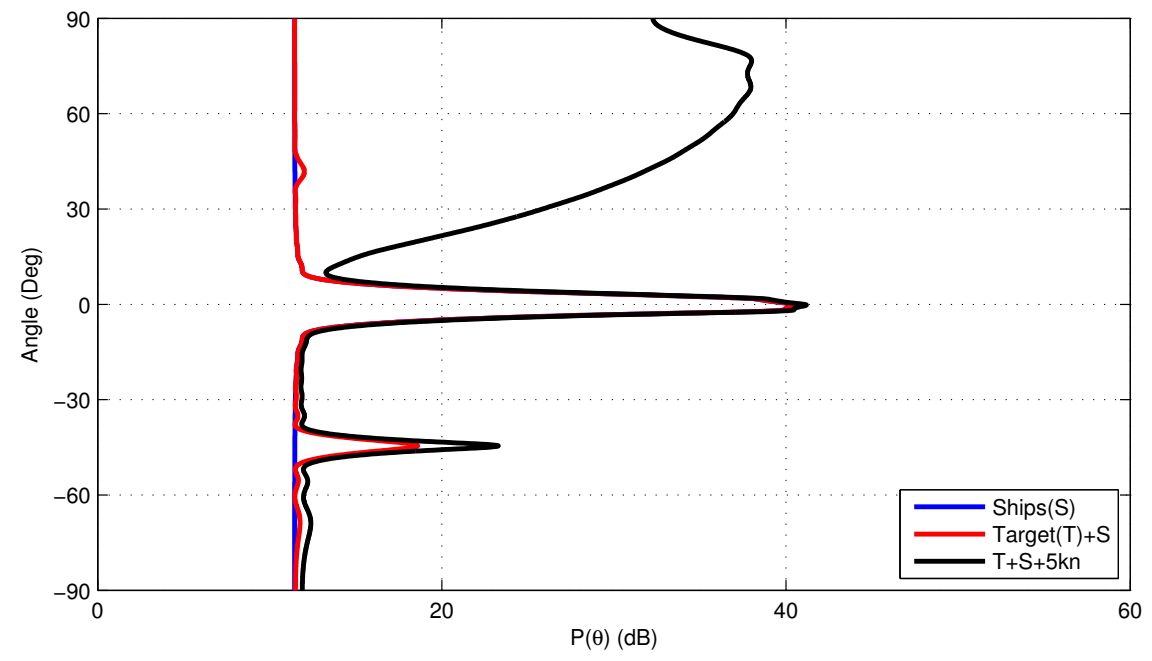

(b)

Figure 4.14: Adaptive beamforming with diagonal loading at $300 \mathrm{~Hz}$. Diagonal loading level is $25 \mathrm{~dB}$. Other parameters are the same as Figure 4.13. 
With these results, we can see that the detection of the target's direct arrival angle is weaker with adaptive beamforming than that with conventional beamforming. One of the possible reasons is the mismatch between assumed steering vector and the actual arrived signal. Another possible reason is that the adaptive beamforming runs out of degrees of freedom. However, the adaptive beamforming could efficiently sharpen the beams. This will provide a favorable detection performance in the presence of nearby interferers.

\subsubsection{Motion Effect Analysis}

This section examines the motion effect on the nearby strong interferers. This simulation includes all the ships (distant ships, mid-range ships and nearby ships) in Church Opal area. The HITS database indicates very few ships near the site of the experiment, as shown in Figure 4.15, which shows the nearest ships are $20 \mathrm{~km}$ away from the VLA, and simulations show that their effect as interferers is minimal.

To create more challenging test for the beamformer, a large tanker at the range of $7 \mathrm{~km}$ is used. This large tanker is the only moving interferer, with a random speed chosen between 15 and $18 \mathrm{kts}$. Because of the efficiency of sharpening beams, adaptive beamforming with $25 \mathrm{~dB}$ diagonal loading is ap-

plied to analyze the target detection performance in the presence of the moving 


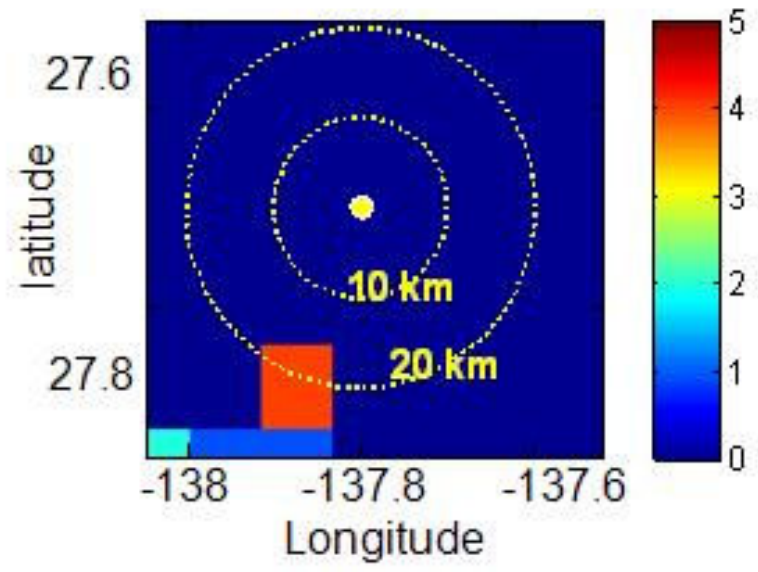

Figure 4.15: Computed nearby ships based on HITS database at the location of Church Opal. The colorbar shows the total ship number in 5000 realizations. For example, at the cell with source to receiver range of $20 \mathrm{~km}$, there are about 4 ships in all during 5000 realizations. The nearby ship based on the realistic data has minimal effects as interferers.

interferer. As we discussed in Section 3.3, we used $\mathrm{L}=60$ snapshots, which is twice of the number of the elements in the array, and the snapshot length between snapshots is 0.1 seconds and 10 seconds.

Figure 4.16 shows the change of source to receiver range during 60 snapshots. The large tanker moves $50.3 \mathrm{~m}$ and $5013.7 \mathrm{~m}$ during the 60 snapshots with the snapshot lengths of 0.1 seconds and 10 seconds, respectively. Note that a snapshot length of 0.1 seconds is not practical because that would result in a coarse frequency resolution, since the mainlobe of the width in the frequency domain is inversely proportional to the snapshot length. Nevertheless, it will be used here to illustrate the impact of that short observation times has 
on the rank of the estimated covariance, if that were possible.

Figure 4.17 shows the eigenvalue spectrum structure of the moving interferer covariance matrix. In Figure $4.17(\mathrm{~b})$, it can be seen that the movement of the large tanker spreads its energy over the eigenvalue spectra, therefore the beamforming output shown next looks like there are several interferers.

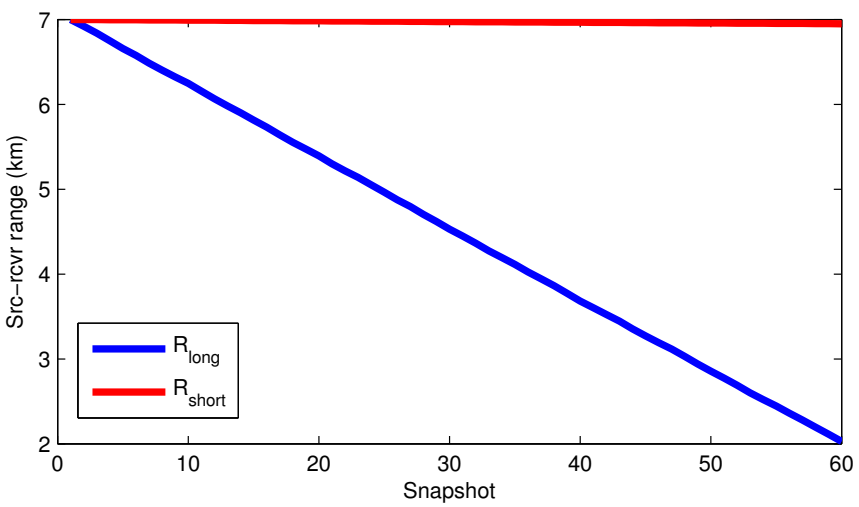

Figure 4.16: Change of source to receiver range during 60 snapshots. The snapshot length are 0.1 seconds and 10 seconds, respectively.

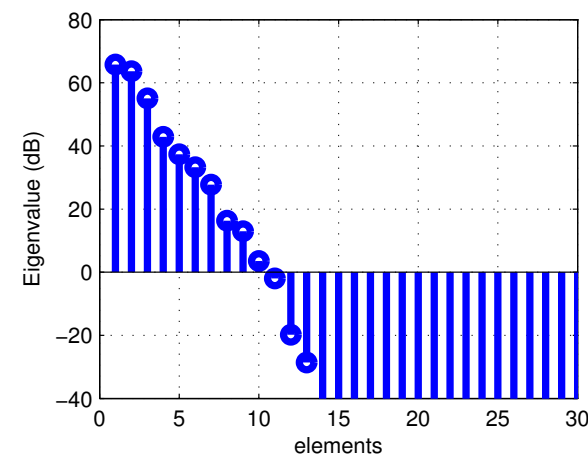

(a)

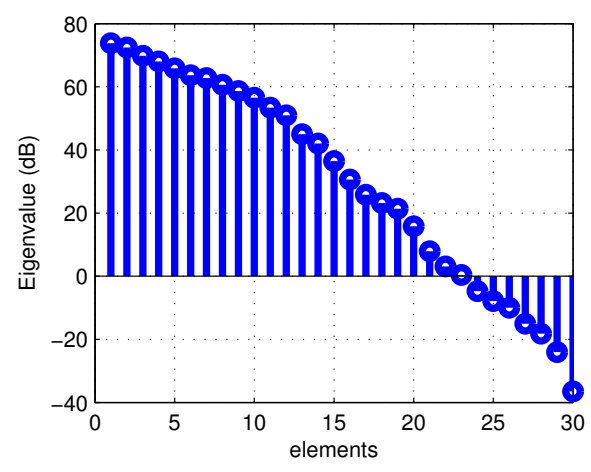

(b)

Figure 4.17: Eigenvalue spectrum structure of the moving interferer covariance matrix. The snapshot length is (a) 0.1 seconds and (b) 10 seconds. 
Figure 4.18 shows the output of the adaptive beamformer with $25 \mathrm{~dB}$ diagonal loading at $300 \mathrm{~Hz}$. The sample covariance matrix is the average over 60 snapshots. The interval time between snapshots are 0.1 seconds and 10 seconds, respectively. In Figure 4.18(a), 5 peaks can be observed at angles of approximately $64,32,-11,-37$, and -64 degrees, respectively. The peak at 64 degrees corresponds to the bottom-surface bounce. The peak at 32 degrees is the direct arrival of the moving large tanker, while the peak at -37 is the corresponding bottom bounce. The peak at -11 degrees is caused by lower order modes which travel very close to horizontal, and the peak at -64 degrees corresponds to the bottom-surface-bottom bounce. In Figure 4.18(b), the target is covered by the interfering noise. The results indicate that using short observation time would minimize the motion effects of interferers. 


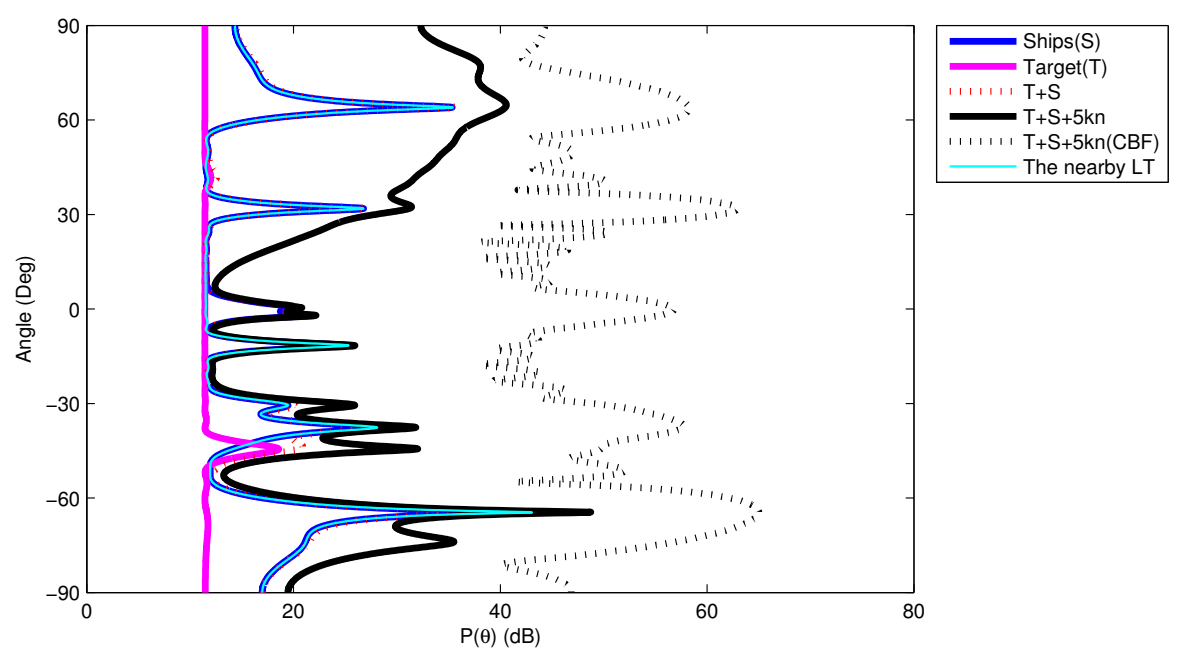

(a)

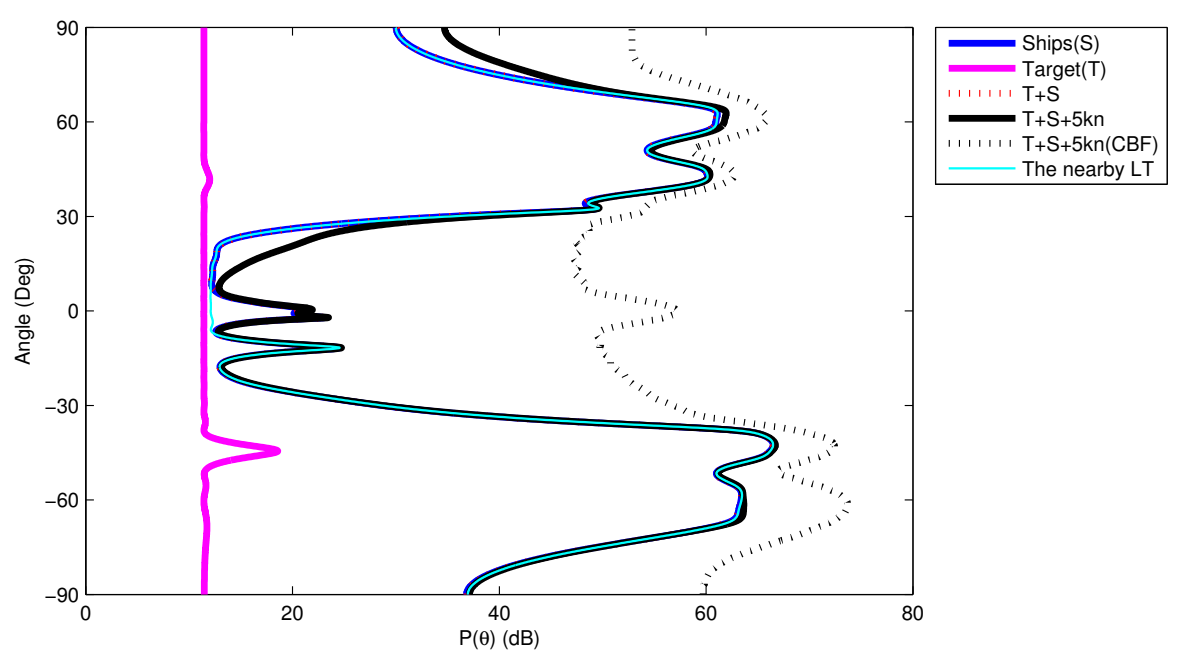

(b)

Figure 4.18: Adaptive beamforming output with $25 \mathrm{~dB}$ diagonal loading at 300 $\mathrm{Hz}$. The target is at $5 \mathrm{~km}$ range, $100 \mathrm{~m}$ depth. Noise includes all shipping noise, $5 \mathrm{kn}$ wind noise, $20 \mathrm{~dB}$ white noise and noise from the moving large tanker. $\mathrm{L}=60$ Snapshots. The interval times between snapshots are (a) 0.1 seconds, and (b) 10 seconds. 


\section{Chapter 5}

\section{Conclusions and Future Work}

A model of ambient noise in deep water channels has been developed to analyze the vertical arrival structure of shipping noise and to separate a target from the ambient noise. Five thousand Monte Carlo simulations were used to obtain a close resemblance of the experimental data provided by HITS database. Parameters for the ocean channel model were chosen in agreement with the experimental observations [1]. Sensitivity to exact parameters of the bottom sediment structure was also explored. The depth dependence of the simulated shipping noise was in agreement with the published experimental measurements of Gaul et al. The results also suggested that the noise from distant shipping (range $>400 \mathrm{~km}$ ) may be significantly reduced when measured on receivers below the critical depth. The ambient noise model was able to calculate noise responses of arrays of hydrophones operating in complicated shipping and environmental conditions. It can be applied to predict directionalities in the deep ocean environments. Furthermore, the VLA produces vertical beams that isolate distant surface ship interference from nearby targets, since the distant shipping noise arrives via shallow grazing angles. Results suggested a favorable detection performance using conventional beamforming with Chebyshev taper 
in the presence of distant shipping interferers and wind speed. The results also illustrated the complex structure of the signal received from both the target and interference, with potentially strong contributions from bottom reflections appearing in the lower beams.

This research also demonstrated the motion effect of interferers by analyzing the eigenvalue spectra. Adaptive beamforming implementing diagonal loading is more useful because the beamwidth is much sharper, which means the ability to isolate target from nearby interferers is much stronger than conventional beamforming. Adaptive beamforming results corresponding to different snapshot times show that motion effects can be minimized by using short observation time.

For the future work, horizontal apertures with adaptive processing can be used to null interference from nearby ships. Tilted array, which can be considered as the combination of vertical and horizontal arrays, can also be explored for adaptive nulling of interference and exploitation of vertical noise structure. Another field for future research is the development of rapid adaptive processing schemes to minimize the source motion effects. The data from NPAL Philippine Sea Experiment 2009 can be used to support quantification of signal and noise structure. 


\section{References}

[1] R. D. Gaul, D. P. Knobles, J. A. Shooter, and A. F. Wittenborn. Ambient noise analysis of deep-ocean measurements in the Northeast Pacific. IEEE J. Ocean. Eng., vol. 32, no. 2, 2007.

[2] F. B. Jensen, W. A. Kuperman, M. B. Porter and H. Schmidt. Computational Ocean Acoustics. AIP series in modern acoustics and signal processing, Springer-Verlag, New York, pp. 279-281, 1993.

[3] L. Brekhovskikh and Yu. Lysanov. Fundamentals of Ocean Acoustics. vol. 8. Springer-Verlag Berlin Heidelberg, 1982, pp. 30.

[4] W. S. Burdic. Underwater Acoustic System Analysis. 2nd ed., Prentice Hall Signal Processing Series, 1991, pp. 280-284.

[5] R. M. Hamson. The modelling of ambient noise due to shipping and wind sources in complex environments. Applied Acoustics, vol. 51, no. 33, pp. 251-287, 1997.

[6] H. Krim and M. Viberg. Two decades of array signal processing research. IEEE Signal Processing Magazine, vol. 13, pp. 67-94, July 1996. 
[7] S. M. Kogon. Experimental results for passive sonar arrays with eigenvector-based adaptive beamformers. 36th Asilomar Conf. Signals, Syst. Computers. vol. 1, pp. 439-447, November 2002.

[8] R. Vincent and O. Besson. Steering vector error and diagonal loading. IEE Proc. -Radar Sonar Navig., vol. 151, no. 6, December 2004.

[9] A. B. Baggeroer and H. Cox. Passive sonar limits upon nulling multiple moving ships with large aperture arrays. Proc. IEEE 33rd Asilomar Conf. Signals, Systems Comput., Pacific Grove, CA, October 1999, pp. 103-108.

[10] R. J. Urick. Principles of Underwater Sound. 3rd ed., McGraw-Hill, New York, 1983.

[11] L.E. Kinsler, A. R. Frey, A. B. Coppens, J. V. Sanders. Fundamentals of Acoustics. 4th ed., John Wiley and Sons, Inc.

[12] M. Porter. The KRAKEN Normal Mode Program, Software available at www.hlsresearch.com

[13] L. Emery, M. Bradley, and T. Hall. Data Base Description (DBD) for the Historical Temporal Shipping Data Base (HITS), Version 4.0. PSI Technical Report TRS-301, October 2001. 
[14] W. M. Renner. Ambient Noise Directionality Estimation System (ANDES) Technical Description. Science Applications Inc. SAIC-86/1645, 1986.

[15] F. Noblesse, G. Delhommeau, H. Y. Kim, and C. Yang. Thin-ship theory and influence of rake and flare. J. of Eng. Mathematics, vol. 64, no.1, 2009.

[16] H. Schmidt. OASES Version 2.2 User Guide and Reference Manual. Department of Ocean Engineering, Massachusetts Institute of Technology, August 1999 .

[17] B.D. Van Veen and K. M. Buckley. Beamforming: a versatile approach to spatial filtering. IEEE ASSP Magazine, April 1988.

[18] L. M. Zurk and M. Velankar. Passive sonar array sub-space processing based on modal decomposition. Oceans 2005 Proceedings of MTS/IEEE, pp. 1-5, September 2005.

[19] A. B. Baggeroer, W. A. Kuperman, and P. N. Mikhalevsky. An overview of matched field methods in ocean acoustics. IEEE J. Ocean. Eng., vol. 18, no. 4, pp. 401-424, October 1993.

[20] H. Cox. Resolving power and sensitivity to mismatch of optimum array processors. J. Acoust. Soc. Am., vol. 54, Issue 3, pp. 771-785, September 1973. 
[21] J. Li and P. Stoica. Robust Adaptive Beamforming. Wiley-Interscience, pp. 57-58, October 2005.

[22] E. L. Hamilton. Geoacoustic modeling of the sea floor. J. Acoust. Soc. Am., Vol. 68, Issue 5, pp. 1313-1340, November 1980.

[23] Z. Li, L. M. Zurk, B. Ma. Vertical Arrival Structure of Shipping Noise in Deep Water Channels. Oceans 2010 Proceedings of MTS/IEEE, Seattle, September 2010.

[24] J. A. Shooter, T. E. DeMary, and A. F. Wittenborn. Depth dependence of noise resulting from ship traffic and wind. IEEE J. Ocean. Eng., vol. 15, no. 4, pp. 292-298, October 1990. 